\title{
Fluid Flows of Mixed Regimes in Porous Media
}

\author{
Emine Celik ${ }^{a}$, Luan Hoang ${ }^{a}$, Akif Ibragimov ${ }^{a}$ and Thinh Kieu ${ }^{b}$ \\ August 8, 2018 \\ ${ }^{a}$ Department of Mathematics and Statistics, Texas Tech University, Box 41042 \\ Lubbock, TX 79409-1042, U. S. A. \\ ${ }^{b}$ Department of Mathematics, University of North Georgia, Gainesville Campus \\ 3820 Mundy Mill Rd., Oakwood, GA 30566, U. S. A. \\ Email addresses: emine.celik@ttu.edu, luan.hoang@ttu.edu, \\ akif.ibraguimov@ttu.edu, thinh.kieu@ung.edu
}

\begin{abstract}
In porous media, there are three known regimes of fluid flows, namely, pre-Darcy, Darcy and post-Darcy. Because of their different natures, these are usually treated separately in literature. To study complex flows when all three regimes may be present in different portions of a same domain, we use a single equation of motion to unify them. Several scenarios and models are then considered for slightly compressible fluids. A nonlinear parabolic equation for the pressure is derived, which is degenerate when the pressure gradient is either small or large. We estimate the pressure and its gradient for all time in terms of initial and boundary data. We also obtain their particular bounds for large time which depend on the asymptotic behavior of the boundary data but not on the initial one. Moreover, the continuous dependence of the solutions on initial and boundary data, and the structural stability for the equation are established.
\end{abstract}

\section{Introduction and the models}

Fluid flows are very common in nature such as in soil, sand, aquifers, oil reservoir, sea ice, plants, bones, etc. Contrary to the usual perception of their simplicity, they, in fact, can be very complicated and are modeled by many different equations of various types. Broadly speaking, they are categorized into three known regimes, namely, pre-Darcy (i.e. pre-linear, non-Darcy), Darcy (linear) and post-Darcy (i.e. post-linear, non-Darcy). While the Darcy regime is well-known, the other two do exist and are studied in physics and engineering. For example, when the Reynolds number is high, there is a deviation from the Darcy law and Forchheimer's equations are usually used to account for it [9, 10, see also [2,20,21]. On the other end of the Reynolds number's range, when it is small, the pre-Darcy regime is observed but not well understood, although it contributes to unexpected oil extraction, see [8,22,23] and references therein.

Concerning mathematical research of fluids in porous media, the flows' diverse nature is much overlooked. Almost all of the papers focus on the Darcy regime which is presented by the (linear) Darcy equation, see e.g. 25]. The post-Darcy regime has been attracted attention recently with the (nonlinear) Forchheimer models, see [1, 5, 6, 12, 15, 18, 24] and references therein. In contrast, the (nonlinear) pre-Darcy regime is virtually ignored. Moreover, the three regimes are always treated separately. This is due to the different natures of the models and the ranges of their applicability. However, this separation is unsatisfactory since the fluid may present all three regimes in different 
unidentified portions of the confinement. Therefore, there is a need to unify the three regimes into one formulation and study the fluid as a whole. This paper aims at deriving admissible models for this unification and analyze their properties mathematically. To the best of our knowledge, this is the first paper to treat such a problem with rigorous mathematics.

We now start the investigation of different types of fluid flows in porous media. Consider fluid flows with velocity $v \in \mathbb{R}^{n}$, pressure $p \in \mathbb{R}$, and density $\rho \in[0, \infty)$. Depending on the range of the Reynolds number, there are different groups of equations to describe their dynamics.

The most popular equation is Darcy's law:

$$
v=-k \nabla p, \text { where } k \text { is a positive constant. }
$$

(In this paper, we will not discuss other variations, such as those of Brinkman-type, for (1.1) or Forchheimer equations (1.3)-(1.5).)

When $|v|$ is small, there are Izbash-type equations that describe the pre-Darcy regime:

$$
|v|^{-\alpha} v=-k \nabla p \quad \text { for some constant power } \alpha \in(0,1) \text { and coefficient } k>0 .
$$

For experimental values of $\alpha$, see e.g. [22,23].

When $|v|$ is large, the following Forchheimer equations are usually used in studying post-Darcy flows.

Forchheimer's two-term law

$$
a v+b|v| v=-\nabla p .
$$

Forchheimer's three-term law

$$
a v+b|v| v+c|v|^{2} v=-\nabla p
$$

Forchheimer's power law

$$
a v+d|v|^{m-1} v=-\nabla p .
$$

Here, the positive numbers $a, b, c, d$, and $m \in(1,2)$ are derived from experiments for each case.

The above three Forchheimer equations can be combined and generalized to the following form:

$$
g_{F}(|v|) v=-\nabla p
$$

where

$$
g_{F}(s)=a_{0}+a_{1} s^{\alpha_{1}}+\cdots+a_{N} s^{\alpha_{N}},
$$

with $N \geq 1, \alpha_{0}=0<\alpha_{1}<\ldots<\alpha_{N}, a_{0}, a_{N}>0, a_{1}, a_{2}, \ldots, a_{N-1} \geq 0$.

The generalized Forchheimer equation (1.6) was intensely used by the authors to model and study fast flows in the porous media (see [1, 3, 6, 11, 15, 18]). The techniques developed in those papers will be essential in our approach and analysis below.

In previous work, each regime pre-Darcy, Darcy, or post-Darcy was studied separately, even though they exist simultaneously in porous media. In particular cases, some models must consider multi-layer domains with each layer having a different regime of fluid flows, see for e.g. section 6.7.8 of [24]. The goal of this section is to model all regimes together in the same domain.

We write a general equation of motion for all cases (1.1)-(1.6) as

$$
\mathbf{G}(v)=-\nabla p,
$$


where $\mathbf{G}$ is a vector field on $\mathbb{R}^{n}$ with $\mathbf{G}(0)=0$. In this paper, based on the known equations (1.2) -(1.6), we study $\mathbf{G}$ of the form

$$
\mathbf{G}(v)= \begin{cases}g(|v|) v & \text { if } v \in \mathbb{R}^{n} \backslash\{0\}, \\ 0 & \text { if } v=0\end{cases}
$$

where $g(s)$ is a continuous function from $(0, \infty)$ to $(0, \infty)$ that satisfies

$$
\lim _{s \searrow 0} s g(s)=0 .
$$

Different forms of $g(s)$ give different models, for example,

$$
g(s)=k^{-1} s^{-\alpha}, k^{-1}, a+b s, a+b s+c s^{2}, a+d s^{m-1}, g_{F}(s),
$$

for equations (1.2), (1.1), (1.3), (1.4), (1.5), (1.6), respectively.

As in our previous work for compressible fluids, to reduce the complexity of the system of equations describing the fluid motion, we solve for velocity $v$ in (1.9) in terms of the pressure gradient $\nabla p$. For example, from (1.2) we have

$$
v=-k^{\frac{1}{1-\alpha}}|\nabla p|^{\frac{\alpha}{1-\alpha}} \nabla p
$$

and from (1.6) we have

$$
v=-K_{F}(|\nabla p|) \nabla p
$$

where

$$
K_{F}(\xi)=\frac{1}{g_{F}\left(G_{F}^{-1}(\xi)\right)} \quad \text { with } G_{F}(s)=s g_{F}(s) \quad \text { for } \xi, s \geq 0 .
$$

Taking the modulus both sides of (1.8), we have

$$
G(|v|)=|\nabla p|,
$$

where

$$
G(s)= \begin{cases}s g(s) & \text { if } s>0 \\ 0 & \text { if } s=0\end{cases}
$$

By (1.10), we have

(g1) $G(s)$ is continuous on $[0, \infty)$.

We assume

(g2) $G(s)$ is strictly increasing on $[0, \infty)$,

(g3) $G(s) \rightarrow \infty$ as $s \rightarrow \infty$, and

(g4) the function $1 / g(s)$ on $(0, \infty)$ can be extended to a continuous function $k_{g}(s)$ on $[0, \infty)$.

By (g1)-(g3), we can invert equation (1.12) to have

$$
|v|=G^{-1}(|\nabla p|) .
$$

Combining this with (g4), we can solve from (1.8) and (1.9) for $v=-k_{g}(|v|) \nabla p$, thus,

$$
v=-K(|\nabla p|) \nabla p,
$$


where

$$
K(\xi)=k_{g}\left(G^{-1}(\xi)\right) \quad \text { for } \xi \geq 0 .
$$

In particular, when $\xi>0$

$$
K(\xi)=\frac{1}{g(s(\xi))} \quad \text { with } \quad s=s(\xi)>0 \quad \text { satisfying } \quad s g(s)=\xi .
$$

One can interpret equation (1.14) as a generalization Darcy equation (1.1) with conductivity $k=K(|\nabla p|)$ depending on the pressure's gradient.

We consider the following two main models. Below, $\mathbf{1}_{E}$ denotes the characteristic (indicator) function of a set $E$.

Model 1. Function $g(s)$ is piece-wise smooth on $(0, \infty)$. Based on (1.2), (1.1) and (1.6) and their validity in different ranges of $|v|$, our first consideration is the following piece-wise defined function

$$
g(s)=\bar{g}(s) \stackrel{\text { def }}{=} c_{1} s^{-\alpha} \mathbf{1}_{\left(0, s_{1}\right)}(s)+c_{2} \mathbf{1}_{\left[s_{1}, s_{2}\right]}(s)+g_{F}(s) \mathbf{1}_{\left(s_{2}, \infty\right)}(s) \quad \text { for } s>0,
$$

where $\alpha \in(0,1)$, and $s_{2}>s_{1}>0$ are fixed threshold values. To avoid abrupt transitions between three regimes, we impose the continuity on $\bar{g}(s)$, that is,

$$
c_{1} s_{1}^{-\alpha}=c_{2}=g_{F}\left(s_{2}\right) .
$$

Note that $\bar{g}(s)$ is not differentiable at $s_{1}, s_{2}$. Obviously, (1.10) holds. Then function $G(s)$ in (1.13) becomes

$$
G(s)=\bar{G}(s) \stackrel{\text { def }}{=} c_{1} s^{1-\alpha} \mathbf{1}_{\left[0, s_{1}\right)}(s)+c_{2} s \mathbf{1}_{\left[s_{1}, s_{2}\right]}(s)+G_{F}(s) \mathbf{1}_{\left(s_{2}, \infty\right)}(s) \quad \text { for } s \geq 0 .
$$

Clearly, conditions (g2)-(g3) are satisfied. Then

$$
\bar{G}^{-1}(\xi)=\left(\frac{\xi}{c_{1}}\right)^{\frac{1}{1-\alpha}} \mathbf{1}_{\left[0, Z_{1}\right)}(\xi)+\frac{\xi}{c_{2}} \mathbf{1}_{\left[Z_{1}, Z_{2}\right]}(\xi)+G_{F}^{-1}(\xi) \mathbf{1}_{\left(Z_{2}, \infty\right)}(\xi) \quad \text { for } \xi \geq 0
$$

where $Z_{1}=c_{2} s_{1}$ and $Z_{2}=c_{2} s_{2}$. Also, (g4) holds true with

$$
k_{\bar{g}}(s)=\frac{s^{\alpha}}{c_{1}} \mathbf{1}_{\left[0, s_{1}\right)}(s)+\frac{1}{c_{2}} \mathbf{1}_{\left[s_{1}, s_{2}\right]}(s)+\frac{1}{g_{F}(s)} \mathbf{1}_{\left(s_{2}, \infty\right)}(s) \quad \text { for } s \geq 0 .
$$

Thus, we derive function $K(\xi)$ in (1.15) explicitly as

$$
K(\xi)=\bar{K}(\xi) \stackrel{\text { def }}{=} M_{1} \xi^{\beta_{1}} \mathbf{1}_{\left[0, Z_{1}\right)}(\xi)+M_{2} \mathbf{1}_{\left[Z_{1}, Z_{2}\right]}(\xi)+K_{F}(\xi) \mathbf{1}_{\left(Z_{2}, \infty\right)}(\xi) \text { for } \xi \geq 0,
$$

where $M_{1}=c_{1}^{-\frac{1}{1-\alpha}}, M_{2}=c_{2}^{-1}$, and

$$
\beta_{1}=\alpha /(1-\alpha)>0 \text {. }
$$

Note that, similar to the function $\bar{g}$, this function $\bar{K}$ is continuous on $[0, \infty)$, continuously differentiable on $(0, \infty) \backslash\left\{Z_{1}, Z_{2}\right\}$.

Model 2. Function $g(s)$ is smooth on $(0, \infty)$. Another generalization is to use a smooth interpolation between pre-Darcy (1.2) and generalized Forchheimer (1.6). Instead of (1.17), we propose the following

$$
g(s)=g_{I}(s) \stackrel{\text { def }}{=} a_{-1} s^{-\alpha}+a_{0}+a_{1} s^{\alpha_{1}}+\cdots+a_{N} s^{\alpha_{N}} \quad \text { for } s>0,
$$


where $N \geq 1, \alpha \in(0,1), \alpha_{N}>0$,

$$
a_{-1}, a_{N}>0 \text { and } a_{i} \geq 0 \quad \forall i=0,1, \ldots, N-1 .
$$

Normally, $a_{0}>0$ and, thus, the model (1.20) already contains the Darcy regime in its formulation. Nonetheless, our mathematical study in this paper allows the case $a_{0}=0$ as well.

If only one function $g_{I}$ is studied, then we can impose $a_{i}>0$ for all $i=-1,0, \ldots, N$. The weaker condition (1.21) is used here to allow comparison between $g_{I}$ functions with different powers $\alpha_{i}$, see section 5 ,

The main advantage of $g_{I}$ over $\bar{g}$ is that it is smooth on $(0, \infty)$. This allows further mathematical analysis of the flows. It also can be used as a framework for perspective interpretation of field data, i.e., matching the coefficients $a_{i}$ for $i=-1,0,1, \ldots, N$ to fit the data.

Similar to Model 1, conditions (1.10) and (g2)-(g4) are satisfied with

$$
\begin{gathered}
G(s)=G_{I}(s) \stackrel{\text { def }}{=} a_{-1} s^{1-\alpha}+a_{0} s+a_{1} s^{1+\alpha_{1}}+\cdots+a_{N} s^{1+\alpha_{N}}, \\
k_{g_{I}}(s)=\frac{s^{\alpha}}{a_{-1}+a_{0} s^{\alpha}+a_{1} s^{\alpha+\alpha_{1}}+\ldots+a_{N} s^{\alpha+\alpha_{N}}}
\end{gathered}
$$

for $s \geq 0$. We then obtain

$$
K(\xi)=K_{I}(\xi) \stackrel{\text { def }}{=} \frac{s(\xi)^{\alpha}}{a_{-1}+a_{0} s(\xi)^{\alpha}+a_{1} s(\xi)^{\alpha+\alpha_{1}}+\ldots+a_{N} s(\xi)^{\alpha+\alpha_{N}}} \quad \text { for } \xi \geq 0
$$

where $s(\xi)=G_{I}^{-1}(\xi)$.

In case we want to consider dependence on the coefficients of $g_{I}(s)$, we denote

$$
\vec{a}=\left(a_{-1}, a_{0}, a_{1}, \ldots, a_{N}\right), \quad g_{I}(s)=g_{I}(s, \vec{a}), \quad \text { and } \quad K_{I}(\xi)=K_{I}(\xi, \vec{a}) .
$$

Model 3. Another way to describe the flows of mixed regimes is to take the formula (1.14) and define the conductivity function $K(\xi)$ directly that possesses some desired properties. In doing so, one can impose the smoothness on $K(\xi)$. An important feature in constructing $K(\xi)$ is to preserve its behavior when $\xi \rightarrow 0$ or $\xi \rightarrow \infty$ to be the same as that of $\bar{K}(\xi)$. As $\xi \rightarrow 0$ it is clear from (1.18) that $\bar{K}(\xi)$ is like $\xi^{\beta_{1}}$. For sufficiently large $\xi$ we have $\bar{K}(\xi)=K_{F}(\xi)$ defined by (1.11). Thus, we recall from Lemma 2.1 of [11] that the function $K_{F}(\xi)$ satisfies

$$
\frac{d_{1}^{-1}}{(1+\xi)^{\beta_{2}}} \leq K_{F}(\xi) \leq \frac{d_{1}}{(1+\xi)^{\beta_{2}}}
$$

where

$$
\beta_{2}=\alpha_{N} /\left(1+\alpha_{N}\right) \in(0,1) \quad \text { and } \quad d_{1}=d_{0}\left(\max \left\{a_{0}, a_{1}, \ldots, a_{N}, a_{0}^{-1}, a_{N}^{-1}\right\}\right)^{1+\beta_{2}}
$$

with $d_{0}>0$ depending on $N$ and $\alpha_{N}$.

In summary, we want $K(\xi)$ to behave like $\xi^{\beta_{1}}$ for small $\xi$, and, as in (1.24), like $(1+\xi)^{-\beta_{2}}$ for large $\xi$. Therefore, ones can introduce

$$
K(\xi)=\hat{K}(\xi) \stackrel{\text { def }}{=} \frac{a \xi^{\beta_{1}}}{\left(1+b \xi^{\beta_{1}}\right)\left(1+c \xi^{\beta_{2}}\right)} \quad \text { for } \xi \geq 0 .
$$

Here, positive coefficients $a, b, c$, and parameters $\beta_{1}, \beta_{2}$ can be used to match experimental or field data. This function $\hat{K}$ belongs to $C^{\infty}((0, \infty))$. 
Model 4. Ones can also refine the model (1.26) to match more accurately $\bar{K}(\xi)$ in (1.18). Specifically, $K(\xi)$ is close to $M_{1} \xi^{\beta_{1}}$ when $\xi \rightarrow 0$, and to $K_{F}(\xi)$ when $\xi \rightarrow \infty$. Then we choose

$$
K(\xi)=K_{M}(\xi) \stackrel{\text { def }}{=} K_{F}(\xi) \cdot \frac{\bar{k} \xi^{\beta_{1}}}{1+\bar{k} \xi^{\beta_{1}}} \quad \text { for } \xi \geq 0,
$$

where $\bar{k}=M_{1} / K_{F}(0)>0$.

Above, we have introduced several models which can be used to interpret experimental and field data. We now use them to investigate the fluid flow's properties. They are used together with other basic equations of continuum mechanics which we recall here.

Continuity equation

$$
\phi \rho_{t}+\nabla \cdot(\rho v)=0
$$

where $\phi \in(0,1)$ is the constant porosity.

Constitutive law for slightly compressible fluids

$$
\frac{d \rho}{d p}=\frac{\rho}{\kappa}
$$

where $1 / \kappa>0$ is small compressibility.

Combining the above two equations with (1.14), we obtain

$$
\left.\phi p_{t}=\kappa \nabla \cdot(K|\nabla p|) \nabla p\right)+K(|\nabla p|)|\nabla p|^{2} .
$$

Since $\kappa$ is large, we neglect the last term in this study. Such a simplification is commonly used in petroleum engineering. The full treatment requires more accurate models for the flows and can use the similar analysis as in [5, 6].

By rescaling $t$, we assume $\kappa=1$ and obtain the following reduced equation

$$
p_{t}=\nabla \cdot(K(|\nabla p|) \nabla p)
$$

where $K$ is $\bar{K}, K_{I}, \hat{K}$ or $K_{M}$.

We will study the initial, boundary value problem (IBVP) associated with the partial differential equation (1.28). We will derive estimates for the solutions, and establish their continuous dependence on the initial and boundary data, and, in case $K=K_{I}$, on the coefficients of the function $g_{I}(s)$ in (1.20). As seen in the next section, the PDE (1.28) is degenerate when either $|\nabla p| \rightarrow 0$ or $|\nabla p| \rightarrow \infty$. Moreover, it possesses a monotonicity of mixed type which requires extra care in the proof and analysis.

The paper is organized as follows. In section 2, we present important properties of $K(\xi)$ including its type of degeneracy (Lemma 2.1) and monotonicity (Lemma 2.4). They are essential not only for the remaining sections 35 in this paper but also for our future work on the models. In section 3, we study solutions of (1.28) subjected to the time-dependent Dirichlet boundary condition $\psi(x, t)$. We derive estimates the $L^{2}$-norm for a solution $p(x, t)$ and the $L^{2-\beta_{2}}$-norm for its gradient, both for all $t \geq 0$ and, particularly, for large $t$, see Theorems 3.3 and 3.7. Furthermore, we show in Theorems 3.4 and 3.8 that if the boundary data is asymptotically small as $t \rightarrow \infty$, then so are these two norms. Section 4 is focused on the continuous dependence of solutions on the initial and boundary data. Theorem 4.2 shows that the difference between two solutions $p_{1}(x, t)$ and $p_{2}(x, t)$ with boundary data $\psi_{1}(x, t)$ and $\psi_{2}(x, t)$, respectively, is small if their initial difference $p_{1}(x, 0)-p_{2}(x, 0)$ and the boundary data's difference $\psi_{1}(x, t)-\psi_{2}(x, t)$ are small, see (4.21). Especially when $t \rightarrow \infty$, the estimates of $p_{1}(x, t)-p_{2}(x, t)$ depend on the asymptotic 
behavior of $\psi_{1}(x, t)-\psi_{2}(x, t)$. In section [5, we consider particularly $g=g_{I}(s, \vec{a}), K=K_{I}(\xi, \vec{a})$ and prove the structural stability of equation (1.28) with respect to the coefficient vector $\vec{a}$ of the function $g_{I}$. In order to obtain this, we first establish in Lemma 5.1 the perturbed monotonicity for our degenerate PDE. It is then proved in Theorem 5.2 that the difference $P(x, t)$ between the two solutions which correspond to two different coefficient vectors $\vec{a}^{(1)}$ and $\vec{a}^{(2)}$ is estimated in terms of their initial difference $P(x, 0)$ and $\left|\vec{a}^{(1)}-\vec{a}^{(2)}\right|$, see (5.14). Moreover, when time goes to infinity, this difference can be controlled by $\left|\vec{a}^{(1)}-\vec{a}^{(2)}\right|$ only, see (5.15).

\section{Basic properties and inequalities}

In this section, we study some properties of the conductivity function $K(\xi)$ which play crucial roles in the analysis of the PDE (1.28). For comparison purpose we define the function

$$
K_{*}(\xi)=\frac{\xi^{\beta_{1}}}{(1+\xi)^{\beta_{1}+\beta_{2}}} \quad \text { for } \xi \geq 0
$$

where $\beta_{1}>0$ and $\beta_{2} \in(0,1)$ are defined in (1.19) and (1.25), respectively.

Let $\xi_{c}=\beta_{1} / \beta_{2}$. It is elementary to see that

(P1) $K_{*}(\xi)$ is increasing on $\left[0, \xi_{c}\right]$,

(P2) $K_{*}(\xi)$ is decreasing on $\left[\xi_{c}, \infty\right)$, and hence,

(P3) $K_{*}\left(\xi_{c}\right)$ is the maximum of $K_{*}(\xi)$ over $[0, \infty)$.

For $m, \xi \geq 0$,

$$
K_{*}(\xi) \xi^{m}=\left(\frac{\xi}{1+\xi}\right)^{\beta_{1}} \frac{\xi^{m}}{(1+\xi)^{\beta_{2}}} \leq \frac{\xi^{m}}{(1+\xi)^{\beta_{2}}} .
$$

Therefore,

$$
K_{*}(\xi) \xi^{m} \leq \xi^{m-\beta_{2}} \quad \forall m \geq 0, \xi \geq 0 .
$$

If $m \geq \beta_{2}$ and $\xi>\delta>0$ then

$$
K_{*}(\xi) \xi^{m}=\left(\frac{\xi}{1+\xi}\right)^{\beta_{1}+\beta_{2}} \xi^{m-\beta_{2}} \geq\left(\frac{\delta}{1+\delta}\right)^{\beta_{1}+\beta_{2}}\left(\xi^{m-\beta_{2}}-\delta^{m-\beta_{2}}\right) .
$$

This inequality is obviously true when $\xi \leq \delta$. Hence,

$$
K_{*}(\xi) \xi^{m} \geq\left(\frac{\delta}{1+\delta}\right)^{\beta_{1}+\beta_{2}}\left(\xi^{m-\beta_{2}}-\delta^{m-\beta_{2}}\right) \quad \forall \delta>0, m \geq \beta_{2}, \xi \geq 0 .
$$

Lemma 2.1. Let $K=\bar{K}, K_{I}, \hat{K}$, and $K_{M}$ as in (1.18), (1.22), (1.26) and (1.27), respectively. Then there exist $d_{2}, d_{3}>0$ such that

$$
d_{2} K_{*}(\xi) \leq K(\xi) \leq d_{3} K_{*}(\xi) \quad \forall \xi \geq 0 .
$$

Consequently, for all $m \geq \beta_{2}$ and $\delta>0$,

$$
d_{2}\left(\frac{\delta}{1+\delta}\right)^{\beta_{1}+\beta_{2}}\left(\xi^{m-\beta_{2}}-\delta^{m-\beta_{2}}\right) \leq K(\xi) \xi^{m} \leq d_{3} \xi^{m-\beta_{2}} \quad \forall \xi \geq 0 .
$$

In particular, when $K=K_{I}$ ones can take

$$
d_{2}=\frac{1}{\max \left\{1, \xi_{0}\right\}^{1+\beta_{1}}} \text { and } d_{3}=\frac{\left(1+\max \left\{1, \beta_{0}\right\}\right)^{\beta_{1}+\beta_{2}}}{\min \left\{1, a_{-1}, a_{N}\right\}^{1+\beta_{1}}}
$$

with $\xi_{0}=a_{-1}+a_{0}+a_{1}+\cdots+a_{N}$. 
Proof. The inequalities in (2.4) clearly holds for $K=\hat{K}$. When $K=K_{M}$, (2.4) can be easily proved by using relation (1.24).

We prove (2.4) for $K=\bar{K}$ now.

For $0 \leq \xi<Z_{1}$, we have

$$
\frac{K(\xi)}{M_{1}\left(1+Z_{1}\right)^{\beta_{1}+\beta_{2}}}=\frac{\xi^{\beta_{1}}}{\left(1+Z_{1}\right)^{\beta_{1}+\beta_{2}}} \leq K_{*}(\xi) \leq \xi^{\beta_{1}}=\frac{K(\xi)}{M_{1}} .
$$

For $Z_{1} \leq \xi \leq Z_{2}$, we have

$$
\frac{Z_{1}^{\beta_{1}} K(\xi)}{M_{2}\left(1+Z_{1}\right)^{\beta_{1}}\left(1+Z_{2}\right)^{\beta_{2}}}=\left(\frac{Z_{1}}{1+Z_{1}}\right)^{\beta_{1}} \frac{1}{\left(1+Z_{2}\right)^{\beta_{2}}} \leq K_{*}(\xi) \leq Z_{2}^{\beta_{1}}=\frac{Z_{2}^{\beta_{1}} K(\xi)}{M_{2}} .
$$

Above, we used, for the first inequality, the fact that the function $x /(x+1)$ is increasing.

For $\xi>Z_{2}$, we have from (1.24) that

$$
\frac{Z_{2}^{\beta_{1}}}{\left(1+Z_{2}\right)^{\beta_{1}}} d_{1}^{-1} K(\xi) \leq \frac{Z_{2}^{\beta_{1}}}{\left(1+Z_{2}\right)^{\beta_{1}}} \cdot \frac{1}{(1+\xi)^{\beta_{2}}} \leq K_{*}(\xi) \leq \frac{1}{(1+\xi)^{\beta_{2}}} \leq d_{1} K(\xi) .
$$

Therefore, relation (2.4) follows (2.7), (2.8), and (2.9).

Next, consider $K=K_{I}$. Since $K_{I}(0)=0$, it suffices to prove (2.4) for $\xi>0$.

Let $s=s(\xi)=G_{I}^{-1}(\xi)>0$. Then we have

$$
\xi=G_{I}(s)=a_{-1} s^{1-\alpha}+a_{0} s+a_{1} s^{1+\alpha_{1}}+\cdots+a_{N} s^{1+\alpha_{N}} .
$$

Note that $\xi_{0}=G_{I}(1)$. We consider the following two cases.

Case 1: $\xi>\xi_{0}$. Then $s>1$ and we have from (2.10) that

$$
a_{N} s^{1+\alpha_{N}} \leq \xi \leq \xi_{0} s^{1+\alpha_{N}} .
$$

This and the fact $K_{I}(\xi)=1 / g_{I}(s)=s / \xi$ give

$$
C_{1} \xi^{-\beta_{2}}=\frac{\left(\xi / \xi_{0}\right)^{\frac{1}{1+\alpha_{N}}}}{\xi} \leq K_{I}(\xi) \leq \frac{\left(\xi / a_{N}\right)^{\frac{1}{1+\alpha_{N}}}}{\xi}=C_{2} \xi^{-\beta_{2}}
$$

where $C_{1}=\xi_{0}^{\beta_{2}-1}$ and $C_{2}=a_{N}^{\beta_{2}-1}$. The first inequality of (2.11) immediately yields the lower bound for $K_{I}(\xi)$ as

$$
K_{I}(\xi) \geq \frac{C_{1}}{(1+\xi)^{\beta_{2}}} \geq \frac{C_{1}}{(1+\xi)^{\beta_{2}}} \cdot \frac{\xi^{\beta_{1}}}{(1+\xi)^{\beta_{1}}}=C_{1} K_{*}(\xi) .
$$

For the upper bound of $K_{I}(\xi)$ we note for $\xi>\xi_{0}$ that

$$
\frac{\xi_{0}}{\xi_{0}+1} \leq \frac{\xi}{\xi+1} \text { and } \xi \geq \frac{\xi+\xi_{0}}{2} \geq \min \left\{1, \xi_{0}\right\} \frac{\xi+1}{2} .
$$

Combining the second inequality of (2.11) and (2.13) gives

$$
K_{I}(\xi) \leq C_{2}\left(\frac{2}{\xi+\xi_{0}}\right)^{\beta_{2}}\left(\frac{\xi_{0}+1}{\xi_{0}} \cdot \frac{\xi}{\xi+1}\right)^{\beta_{1}} \leq C_{3} \frac{\xi^{\beta_{1}}}{(\xi+1)^{\beta_{1}+\beta_{2}}}=C_{3} K_{*}(\xi),
$$

where

$$
C_{3}=C_{2}\left(\frac{\xi_{0}+1}{\xi_{0}}\right)^{\beta_{1}}\left(\frac{2}{\min \left\{1, \xi_{0}\right\}}\right)^{\beta_{2}}
$$


Case 2: $0<\xi \leq \xi_{0}$. We have $0<s \leq 1$ in this case and

$$
\frac{s^{\alpha}}{\xi_{0}} \leq K_{I}(\xi)=\frac{s^{\alpha}}{a_{-1}+a_{0} s^{\alpha_{1}+\alpha}+\cdots+a_{N} s^{\alpha_{N}+\alpha}} \leq \frac{s^{\alpha}}{a_{-1}} .
$$

By (2.10),

$$
a_{-1} s^{1-\alpha} \leq \xi \leq \xi_{0} s^{1-\alpha} \text { which implies }\left(\xi / \xi_{0}\right)^{\frac{1}{1-\alpha}} \leq s \leq\left(\xi / a_{-1}\right)^{\frac{1}{1-\alpha}} .
$$

Utilizing this in (2.15) yields

$$
\frac{\xi^{\beta_{1}}}{\xi_{0}^{1+\beta_{1}}} \leq K_{I}(\xi) \leq \frac{\xi^{\beta_{1}}}{a_{-1}^{1+\beta_{1}}}
$$

Since $\xi \leq \xi_{0}$, we obtain

$$
C_{4} \frac{\xi^{\beta_{1}}}{(1+\xi)^{\beta_{1}+\beta_{2}}} \leq K_{I}(\xi) \leq \frac{1}{a_{-1}^{1+\beta_{1}}} \xi^{\beta_{1}} \frac{\left(1+\xi_{0}\right)^{\beta_{1}+\beta_{2}}}{(1+\xi)^{\beta_{1}+\beta_{2}}}=C_{5} \frac{\xi^{\beta_{1}}}{(1+\xi)^{\beta_{1}+\beta_{2}}},
$$

where $C_{4}=1 / \xi_{0}^{1+\beta_{1}}$ and $C_{5}=\left(1+\xi_{0}\right)^{\beta_{1}+\beta_{2}} / a_{-1}^{1+\beta_{1}}$. Combining inequalities (2.12), (2.14) and (2.16), we have for both cases that

$$
C_{6} K_{*}(\xi) \leq K_{I}(\xi) \leq C_{7} K_{*}(\xi),
$$

where $C_{6}=\min \left\{C_{1}, C_{4}\right\}$ and $C_{7}=\max \left\{C_{3}, C_{5}\right\}$.

Note that

$$
C_{6}=\frac{1}{\max \left\{\xi_{0}^{1+\beta_{1}}, \xi_{0}^{1-\beta_{2}}\right\}} \geq \frac{1}{\max \left\{1, \xi_{0}\right\}^{1+\beta_{1}}}=d_{2}
$$

and

$$
\begin{aligned}
C_{7} & =\max \left\{\left(\frac{\xi_{0}+1}{\xi_{0}}\right)^{\beta_{1}} \frac{2^{\beta_{2}}}{a_{N}^{1-\beta_{2}} \min \left\{1, \xi_{0}\right\}^{\beta_{2}}}, \frac{\left(1+\xi_{0}\right)^{\beta_{1}+\beta_{2}}}{a_{-1}^{1+\beta_{1}}}\right\} \\
& \leq \max \left\{\frac{\left(\xi_{0}+1\right)^{\beta_{1}} 2^{\beta_{2}}}{\left(\min \left\{1, a_{-1}, a_{N}\right\}\right)^{\beta_{1}+\left(1-\beta_{2}\right)+\beta_{2}}}, \frac{\left(1+\xi_{0}\right)^{\beta_{1}+\beta_{2}}}{\left(\min \left\{1, a_{-1}, a_{N}\right\}\right)^{1+\beta_{1}}}\right\} \\
& \leq \frac{\left(1+\max \left\{1, \xi_{0}\right\}\right)^{\beta_{1}+\beta_{2}}}{\min \left\{1, a_{-1}, a_{N}\right\}^{1+\beta_{1}}}=d_{3} .
\end{aligned}
$$

Hence we obtain (2.4) from (2.17).

Combining (2.4) with (2.2) and (2.3) gives (2.5). The proof is complete.

Combining (2.4) and (P3) gives the upper bound for $K(\xi)$ as

$$
K(\xi) \leq d_{4} \stackrel{\text { def }}{=} d_{3} K_{*}\left(\xi_{c}\right) \quad \forall \xi \in[0, \infty) .
$$

Lemma 2.2. Let $K=K_{I}, \hat{K}$, and $K_{M}$ as in (1.22), (1.26), and (1.27), respectively. Then for all $\xi>0$ one has

$$
-\beta_{2} \frac{K(\xi)}{\xi} \leq K^{\prime}(\xi) \leq \beta_{1} \frac{K(\xi)}{\xi}
$$

and, consequently,

$$
\left|K^{\prime}(\xi)\right| \leq \max \left\{\beta_{1}, \beta_{2}\right\} \frac{K(\xi)}{\xi} .
$$

In case $K=\bar{K}$ in (1.18), the inequalities (2.19) and (2.20) hold for $0<\xi \neq Z_{1}, Z_{2}$. 
Proof. Let $\xi>0$, then $s(\xi)=G^{-1}(\xi)>0$. First, consider $g=g_{I}$ and $K=K_{I}$. By the chain rule, we have from (1.16) that

$$
K^{\prime}(\xi)=-\frac{g^{\prime}(s(\xi)) s^{\prime}(\xi)}{g(s(\xi))^{2}}
$$

Denote $s=s(\xi)$, then $s \cdot g(s)=\xi$. Hence, $s^{\prime} g(s)+s g^{\prime}(s) s^{\prime}=1$ which yields

$$
s^{\prime}(\xi)=\frac{1}{g(s)+s g^{\prime}(s)} .
$$

Substituting (2.22) into (2.21) yields

$$
\begin{aligned}
K^{\prime}(\xi) & =-\frac{g^{\prime}(s)}{g(s)^{2}} \cdot \frac{1}{g(s)+s g^{\prime}(s)}=-\frac{1}{g(s)} \cdot \frac{1}{s g(s)} \cdot \frac{g^{\prime}(s) s}{g(s)+s g^{\prime}(s)} \\
& =-\frac{K(\xi)}{\xi} \cdot \frac{g^{\prime}(s) s}{g(s)+s g^{\prime}(s)}=\frac{K(\xi)}{\xi}\left(\frac{g(s)}{g(s)+s g^{\prime}(s)}-1\right) .
\end{aligned}
$$

Now, for any $s>0$, ones observe that

$$
g(s)+s g^{\prime}(s)=(1-\alpha) a_{-1} s^{-\alpha}+a_{0}+\sum_{i=1}^{N} a_{i}\left(\alpha_{i}+1\right) s^{\alpha_{i}}
$$

and, by the fact $0<1-\alpha<1+\alpha_{i}<1+\alpha_{N}$, have

$$
(1-\alpha) g(s) \leq g(s)+s g^{\prime}(s) \leq\left(1+\alpha_{N}\right) g(s)
$$

Then

$$
-\beta_{2}=\frac{1}{1+\alpha_{N}}-1 \leq \frac{g(s)}{g(s)+s g^{\prime}(s)}-1 \leq \frac{1}{1-\alpha}-1=\beta_{1} .
$$

Therefore, inequality (2.19) follows this and (2.23).

If $K=\bar{K}$ then

$$
\bar{K}^{\prime}(\xi)= \begin{cases}M_{1} \beta_{1} \xi^{\beta_{1}-1}=\beta_{1} \bar{K}(\xi) / \xi, & \text { if } 0<\xi<Z_{1}, \\ 0, & \text { if } Z_{1}<\xi<Z_{2}, \\ K_{F}^{\prime}(\xi), & \text { if } \xi>Z_{2} .\end{cases}
$$

We recall from [1] that

$$
-\beta_{2} \frac{K_{F}(\xi)}{\xi} \leq K_{F}^{\prime}(\xi) \leq 0
$$

Then the relation (2.19) obviously follows (2.24) and (2.25) for $0<\xi \neq Z_{1}, Z_{2}$.

If $K=\hat{K}$ then

$$
\frac{\beta_{1}}{\xi} \geq \frac{K^{\prime}}{K}=(\ln K)^{\prime}=\frac{\beta_{1}}{\xi}-\frac{b \beta_{1} \xi^{\beta_{1}-1}}{1+b \xi^{\beta_{1}}}-\frac{c \beta_{2} \xi^{\beta_{2}-1}}{1+c \xi^{\beta_{2}}} \geq-\frac{\beta_{2}}{\xi} \frac{c \xi^{\beta_{2}}}{1+c \xi^{\beta_{2}}} \geq-\frac{\beta_{2}}{\xi} .
$$

This leads to (2.19).

Finally, consider $K=K_{M}$. Write $K_{M}(\xi)=K_{F}(\xi) M(\xi)$, where $M(\xi)=\bar{k} \xi^{\beta_{1}} /\left(1+\bar{k} \xi^{\beta_{1}}\right)$.

On the one hand, $M^{\prime}(\xi) \geq 0$, hence

$$
K_{M}^{\prime}(\xi)=K_{F}^{\prime}(\xi) M(\xi)+K_{F}(\xi) M^{\prime}(\xi) \geq K_{F}^{\prime}(\xi) M(\xi) \geq-\beta_{2} \frac{K_{F}(\xi) M(\xi)}{\xi}=-\beta_{2} \frac{K_{M}(\xi)}{\xi} .
$$


On the other hand, $K_{F}^{\prime}(\xi) \leq 0$ by (2.25), and

$$
M^{\prime}(\xi)=M(\xi)\left(\frac{\beta_{1}}{\xi}-\frac{\bar{k} \beta_{1} \xi^{\beta_{1}-1}}{1+\bar{k} \xi^{\beta_{1}}}\right) \leq \frac{\beta_{1}}{\xi} M(\xi),
$$

hence

$$
K_{M}^{\prime}(\xi)=K_{F}^{\prime}(\xi) M(\xi)+K_{F}(\xi) M^{\prime}(\xi) \leq K_{F}(\xi) M^{\prime}(\xi) \leq \frac{\beta_{1}}{\xi} K_{F}(\xi) M(\xi)=\beta_{1} \frac{K_{M}(\xi)}{\xi} .
$$

The proof is complete.

Corollary 2.3. Let $K=\bar{K}, K_{I}, \hat{K}, K_{M}$. Then the function $\xi^{m} K(\xi)$ is increasing on $[0, \infty)$ for any real number $m \geq \beta_{2}$.

Proof. First, consider $K=K_{I}, \hat{K}$, or $K_{M}$. According to (2.19),

$$
\left(\xi^{m} K(\xi)\right)^{\prime}=m \xi^{m-1} K(\xi)+\xi^{m} K^{\prime}(\xi) \geq m \xi^{m-1} K(\xi)-\beta_{2} \xi^{m-1} K(\xi) \geq 0
$$

for any $\xi>0$. Hence $\xi^{m} K(\xi)$ is increasing on $[0, \infty)$. If $K=\bar{K}$, then the statement is true on intervals not having $Z_{1}$ or $Z_{2}$ as an interior point. By continuity of $\xi^{m} \bar{K}(\xi)$ on $[0, \infty)$, the statement then holds true on $[0, \infty)$.

Lemma 2.4 (Monotonicity). Let $K=\bar{K}, K_{I}, \hat{K}, K_{M}$, then

$$
\left(K\left(\left|y^{\prime}\right|\right) y^{\prime}-K(|y|) y\right) \cdot\left(y^{\prime}-y\right) \geq \frac{d_{5}\left|y-y^{\prime}\right|^{2+\beta_{1}}}{\left(1+|y|+\left|y^{\prime}\right|\right)^{\beta_{1}+\beta_{2}}} \quad \forall y, y^{\prime} \in \mathbb{R}^{n}
$$

where

$$
d_{5}=\frac{d_{2}\left(1-\beta_{2}\right)}{2^{\beta_{1}+1}\left(\beta_{1}+1\right)} .
$$

Proof. Let $y \neq y^{\prime}$ and denote by $\left[y, y^{\prime}\right]$ the line segment connecting $y$ and $y^{\prime}$.

Case 1: The origin does not belong to $\left[y, y^{\prime}\right]$. We parametrize $\left[y, y^{\prime}\right]$ by

$$
\gamma(t)=t y^{\prime}+(1-t) y \quad \text { for } t \in[0,1] .
$$

Define

$$
h(t)=K(|\gamma(t)|) \gamma(t) \cdot\left(y^{\prime}-y\right) \quad \text { for } t \in[0,1] .
$$

In case $K=K_{I}, \hat{K}, K_{M}$, function $h(t) \in C^{1}([0,1])$. When $K=\bar{K}, h(t)$ is continuous on $[0,1]$ and $h^{\prime}(t)$ is piecewise continuous on $[0,1]$ with at most four points of jump discontinuity at which $|\gamma(t)|=Z_{1}$ or $Z_{2}$. By fundamental theorem of calculus,

$$
I \stackrel{\text { def }}{=}\left[K\left(\left|y^{\prime}\right|\right) y^{\prime}-K(|y|) y\right] \cdot\left(y^{\prime}-y\right)=h(1)-h(0)=\int_{0}^{1} h^{\prime}(t) d t .
$$

At $t$ where $h^{\prime}(t)$ exists, we calculate

$$
h^{\prime}(t)=K(|\gamma(t)|)\left|y^{\prime}-y\right|^{2}+K^{\prime}(|\gamma(t)|) \frac{\left|\gamma(t) \cdot\left(y^{\prime}-y\right)\right|^{2}}{|\gamma(t)|} .
$$


By (2.19) and Cauchy-Schwarz inequality

$$
\begin{aligned}
h^{\prime}(t) & \geq K(|\gamma(t)|)\left|y^{\prime}-y\right|^{2}-\beta_{2} \frac{K(|\gamma(t)|)}{|\gamma(t)|} \frac{\left|\gamma(t) \cdot\left(y^{\prime}-y\right)\right|^{2}}{|\gamma(t)|} \\
& \geq K(|\gamma(t)|)\left|y^{\prime}-y\right|^{2}-\beta_{2} \frac{K(|\gamma(t)|)}{|\gamma(t)|} \frac{|\gamma(t)|^{2}\left|y^{\prime}-y\right|^{2}}{|\gamma(t)|}=\left(1-\beta_{2}\right) K(|\gamma(t)|)\left|y^{\prime}-y\right|^{2} .
\end{aligned}
$$

Applying Lemma 2.1 and triangle inequality $|\gamma(t)| \leq|y|+\left|y^{\prime}\right|$, we infer

$$
h^{\prime}(t) \geq\left(1-\beta_{2}\right)\left|y^{\prime}-y\right|^{2} \frac{d_{2}|\gamma(t)|^{\beta_{1}}}{(1+|\gamma(t)|)^{\beta_{1}+\beta_{2}}} \geq \frac{d_{2}\left(1-\beta_{2}\right)\left|y^{\prime}-y\right|^{2}}{\left(1+|y|+\left|y^{\prime}\right|\right)^{\beta_{1}+\beta_{2}}}|\gamma(t)|^{\beta_{1}} .
$$

Together with (2.28), it implies

$$
I \geq \frac{d_{2}\left(1-\beta_{2}\right)\left|y^{\prime}-y\right|^{2}}{\left(1+|y|+\left|y^{\prime}\right|\right)^{\beta_{1}+\beta_{2}}} \int_{0}^{1}|\gamma(t)|^{\beta_{1}} d t .
$$

It remains to estimate the last integral. Let $z_{0}=\frac{y^{\prime}+y}{2\left|y^{\prime}-y\right|}$ and $u=\frac{y^{\prime}-y}{\left|y^{\prime}-y\right|}$. Note that $|u|=1$. Then we write

$$
\begin{aligned}
\int_{0}^{1}|\gamma(t)|^{\beta_{1}} d t & =\left|y^{\prime}-y\right|^{\beta_{1}} \int_{0}^{1}\left|z_{0}+\left(t-\frac{1}{2}\right) u\right|^{\beta_{1}} d t \\
& =\left|y^{\prime}-y\right|^{\beta_{1}} \int_{0}^{1}\left(\left|z_{0}\right|^{2}+2\left(t-\frac{1}{2}\right) z_{0} \cdot u+\left(t-\frac{1}{2}\right)^{2}\right)^{\beta_{1} / 2} d t .
\end{aligned}
$$

If $z_{0} \cdot u \geq 0$ then

$$
\int_{0}^{1}|\gamma(t)|^{\beta_{1}} d t \geq\left|y^{\prime}-y\right|^{\beta_{1}} \int_{1 / 2}^{1}\left(t-\frac{1}{2}\right)^{\beta_{1}} d t=\frac{\left|y^{\prime}-y\right|^{\beta_{1}}}{2^{\beta_{1}+1}\left(\beta_{1}+1\right)} .
$$

If $z_{0} \cdot u<0$ then

$$
\int_{0}^{1}|\gamma(t)|^{\beta_{1}} d t \geq\left|y^{\prime}-y\right|^{\beta_{1}} \int_{0}^{1 / 2}\left(\frac{1}{2}-t\right)^{\beta_{1}} d t=\frac{\left|y^{\prime}-y\right|^{\beta_{1}}}{2^{\beta_{1}+1}\left(\beta_{1}+1\right)} .
$$

In both cases, we have

$$
I \geq \frac{d_{2}\left(1-\beta_{2}\right)}{2^{\beta_{1}+1}\left(\beta_{1}+1\right)} \cdot \frac{\left|y^{\prime}-y\right|^{\beta_{1}+2}}{\left(1+|y|+\left|y^{\prime}\right|\right)^{\beta_{1}+\beta_{2}}}
$$

which proves (2.27).

Case 2: The origin belongs to $\left[y, y^{\prime}\right]$. We replace $y^{\prime}$ by some $y_{\epsilon} \neq 0$ such that $0 \notin\left[y, y_{\epsilon}\right]$, and $y_{\epsilon} \rightarrow y^{\prime}$ as $\epsilon \rightarrow 0$. Then let apply the inequality established in Case 1 for $y$ and $y_{\epsilon}$, then let $\epsilon \rightarrow 0$.

Remark 2.5. Our proof of (2.33) from (2.30) simplifies DiBenedetto's arguments in [7], p. 13, 14.

Degree Condition: One of the following equivalent conditions

$$
\operatorname{deg}(g) \leq \frac{4}{n-2}, \quad \beta_{2} \leq \frac{4}{n+2}, \quad 2 \leq\left(2-\beta_{2}\right)^{*}=\frac{\left(2-\beta_{2}\right) n}{n-2+\beta_{2}}, \quad 2-\beta_{2} \geq \frac{2 n}{n+2} .
$$

(Above, $\left(2-\beta_{2}\right)^{*}$ is the Sobolev exponent corresponding to $2-\beta_{2}$.) 
Hereafter, we assume the Degree Condition. Then the Sobolev space $W^{1,2-\beta_{2}}(U)$ is continuously embedded into $L^{2}(U)$. Also, the Poincaré-Sobolev inequality

$$
\|u\|_{L^{2}(U)} \leq C_{\mathrm{PS}}\|\nabla u\|_{L^{2-\beta_{2}(U)}}
$$

holds for all functions $u \in W^{1,2-\beta_{2}}(U)$ which vanish on the boundary $\Gamma$, where $C_{\mathrm{PS}}$ is a positive constant.

In statements and calculations throughout, we use short-hand writing $\|\cdot\|=\|\cdot\|_{L^{2}(U)}$ and $\|u(t)\|_{L^{p}}=\|u(\cdot, t)\|_{L^{p}(U)}$ for a function $u(x, t)$ of $x$ and $t$.

\section{The IBVP and estimates of its solutions}

Let $K(\xi)$ be one of the functions $\bar{K}(\xi), K_{I}(\xi), \hat{K}(\xi), K_{M}(\xi)$. Consider the following IBVP for the main PDE (1.28):

$$
\begin{cases}p_{t}=\nabla \cdot(K(|\nabla p|) \nabla p) & \text { in } U \times(0, \infty), \\ p(x, 0)=p_{0}(x), & \text { in } U \\ p=\psi(x, t), & \text { on } \partial U \times(0, \infty) .\end{cases}
$$

Dealing with the boundary condition, let $\Psi(x, t)$ be an extension of $\psi$ from $x \in \Gamma$ to $x \in \bar{U}$.

Let $\bar{p}=p-\Psi$. Then

$$
\begin{cases}\bar{p}_{t}=\nabla \cdot(K(|\nabla p|) \nabla p)-\Psi_{t} & \text { in } U \times(0, \infty), \\ \bar{p}(x, 0)=p_{0}(x)-\Psi(x, 0), & \text { in } U \\ \bar{p}=0, & \text { on } \partial U \times(0, \infty) .\end{cases}
$$

We will focus on estimates for $\bar{p}(x, t)$. The estimates for $p(x, t)$ can be obtained by simply using the triangle inequality

$$
|p(x, t)| \leq|\bar{p}(x, t)|+|\Psi(x, t)| .
$$

Also, our results are stated in terms of $\Psi(x, t)$. These can be rewritten in terms of $\psi(x, t)$ by using a specific extension. For instance, the harmonic extension is utilized in [11] with the use of norm relations in [19].

Throughout the paper, we will frequently use the following basic inequalities. By Young's inequality, we have

$$
\begin{gathered}
x^{\beta} \leq x^{\gamma_{1}}+x^{\gamma_{2}} \quad \text { for all } x>0, \gamma_{1} \leq \beta \leq \gamma_{2}, \\
x^{\beta} \leq 1+x^{\gamma} \quad \text { for all } x \geq 0, \gamma \geq \beta>0 .
\end{gathered}
$$

For any $r \geq 1, x_{1}, x_{2}, \ldots, x_{k} \geq 0$, and $a, b \in \mathbb{R}^{n}$,

$$
\begin{gathered}
\left(x_{1}+x_{2}+\ldots+x_{k}\right)^{r} \leq k^{r-1}\left(x_{1}^{r}+x_{2}^{r}+\ldots+x_{k}^{r}\right), \\
|a-b|^{r} \geq 2^{1-r}|a|^{r}-|b|^{r} .
\end{gathered}
$$

We also recall here a useful inequality from [13.

Definition 3.1. Given a function $f(t)$ defined on $I=[0, \infty)$. We denote by $E n v(f)$ a continuous, increasing function $F(t)$ on $I$ such that $F(t) \geq f(t)$ for all $t \in I$. 
Lemma 3.2 ( [13], Lemma 2.7). Let $\theta>0$ and let $y(t) \geq 0, h(t)>0, f(t) \geq 0$ be continuous functions on $[0, \infty)$ that satisfy

$$
y^{\prime}(t) \leq-h(t) y(t)^{\theta}+f(t) \quad \text { for all } t>0 .
$$

Then

$$
y(t) \leq y(0)+[\operatorname{Env}(f(t) / h(t))]^{\frac{1}{\theta}} \text { for all } t \geq 0
$$

If $\int_{0}^{\infty} h(t) d t=\infty$ then

$$
\limsup _{t \rightarrow \infty} y(t) \leq \limsup _{t \rightarrow \infty}[f(t) / h(t)]^{\frac{1}{\theta}}
$$

Notation for constants. In this section and section 4 below, the symbol $C$ denotes a generic positive constant independent of the initial and boundary data; it may depend on the function $g(s)$ and the Poincaré-Sobolev constant $C_{P S}$ in (2.34). In a particular proof, $C_{0}, C_{1}, \ldots$ denote positive constants of this type but having their values fixed.

\subsection{Energy estimates}

In this subsection, we obtain $L^{2}$-estimates for the solution $p(x, t)$ for all time $t \geq 0$ and for $t \rightarrow \infty$.

Theorem 3.3. There exists a positive constant $C$ such that for all $t \geq 0$,

$$
\|\bar{p}(t)\|^{2} \leq\|\bar{p}(0)\|^{2}+C[1+\operatorname{Env} f(t)]^{\frac{2}{2-\beta_{2}}},
$$

where

$$
f(t)=f[\Psi](t) \stackrel{\text { def }}{=}\|\nabla \Psi(t)\|^{2}+\left\|\Psi_{t}(t)\right\|^{\frac{2-\beta_{2}}{1-\beta_{2}}}
$$

Furthermore,

$$
\limsup _{t \rightarrow \infty}\|\bar{p}(t)\|^{2} \leq C\left(1+\limsup _{t \rightarrow \infty} f(t)\right)^{\frac{2}{2-\beta_{2}}} .
$$

Proof. Multiplying both sides of first equation in (3.2) by $\bar{p}$, integrating over the domain $U$ and using integration by parts we find that

$$
\begin{aligned}
\frac{1}{2} \frac{d}{d t}\|\bar{p}\|^{2} & =-\int_{U} K(|\nabla p|) \nabla p \cdot \nabla \bar{p} d x-\int_{U} \Psi_{t} \bar{p} d x \\
& =-\int_{U} K(|\nabla p|)|\nabla p|^{2} d x+\int_{U} K(|\nabla p|) \nabla p \cdot \nabla \Psi d x-\int_{U} \Psi_{t} \bar{p} d x .
\end{aligned}
$$

Using Cauchy's inequality and the bound (2.18) for function $K(\cdot)$, we obtain

$$
\begin{aligned}
\int_{U} K(|\nabla p|) \nabla p \cdot \nabla \Psi d x & \leq \frac{1}{2} \int_{U} K(|\nabla p|)|\nabla p|^{2} d x+\frac{1}{2} \int_{U} K(|\nabla p|)|\nabla \Psi|^{2} d x \\
& \leq \frac{1}{2} \int_{U} K(|\nabla p|)|\nabla p|^{2} d x+C\|\nabla \Psi\|^{2} .
\end{aligned}
$$

Let $\varepsilon>0$. By Hölder's and Young's inequalities,

$$
-\int_{U} \Psi_{t} \bar{p} d x \leq\|\bar{p}\|\left\|\Psi_{t}\right\| \leq \varepsilon\|\bar{p}\|^{2-\beta_{2}}+C \varepsilon^{-\frac{1}{1-\beta_{2}}}\left\|\Psi_{t}\right\|^{\frac{2-\beta_{2}}{1-\beta_{2}}} .
$$

Therefore,

$$
\frac{d}{d t}\|\bar{p}\|^{2} \leq-\int_{U} K(|\nabla p|)|\nabla p|^{2} d x+2 \varepsilon\|\bar{p}\|^{2-\beta_{2}}+C\|\nabla \Psi\|^{2}+C \varepsilon^{-\frac{1}{1-\beta_{2}}}\left\|\Psi_{t}\right\|^{\frac{2-\beta_{2}}{1-\beta_{2}}} .
$$


Let $\delta \in(0,1]$. By virtue of (2.5) and applying (3.6) to $r=2-\beta_{2}, a=\nabla \bar{p}, b=-\nabla \Psi$, we have

$$
\begin{aligned}
K(|\nabla p|)|\nabla p|^{2} & \geq d_{2}\left(\frac{\delta}{1+\delta}\right)^{\beta_{1}+\beta_{2}}\left(|\nabla p|^{2-\beta_{2}}-\delta^{2-\beta_{2}}\right) \\
& \geq d_{2}\left(\frac{\delta}{1+\delta}\right)^{\beta_{1}+\beta_{2}}\left(2^{\beta_{2}-1}|\nabla \bar{p}|^{2-\beta_{2}}-|\nabla \Psi|^{2-\beta_{2}}-\delta^{2-\beta_{2}}\right) \\
& \geq \frac{d_{2} \delta^{\beta_{1}+\beta_{2}}}{2^{\beta_{1}+1}}|\nabla \bar{p}|^{2-\beta_{2}}-d_{2}|\nabla \Psi|^{2-\beta_{2}}-d_{2} \delta^{2+\beta_{1}} .
\end{aligned}
$$

Hence, we obtain

$$
\begin{aligned}
& \frac{d}{d t}\|\bar{p}\|^{2} \leq-\frac{d_{2} \delta^{\beta_{1}+\beta_{2}}}{2^{\beta_{1}+1}} \int_{U}|\nabla \bar{p}|^{2-\beta_{2}} d x+C \int_{U}|\nabla \Psi|^{2-\beta_{2}} d x+C \delta^{2+\beta_{1}} \\
& +2 \varepsilon\|\bar{p}\|^{2-\beta_{2}}+C\|\nabla \Psi\|^{2}+C \varepsilon^{-\frac{1}{1-\beta_{2}}}\left\|\Psi_{t}\right\|^{\frac{2-\beta_{2}}{1-\beta_{2}}} .
\end{aligned}
$$

Using Poincaré-Sobolev's inequality (2.34), we bound $\int_{U}|\nabla \bar{p}|^{2-\beta_{2}} d x$ from below by

$$
\int_{U}|\nabla \bar{p}|^{2-\beta_{2}} d x \geq \frac{\|\bar{p}\|^{2-\beta_{2}}}{C_{\mathrm{PS}}^{2-\beta_{2}}}
$$

For comparison of $\nabla \Psi$-terms on the right-hand side of (3.13), applying Hölder's inequality gives

$$
\int_{U}|\nabla \Psi|^{2-\beta_{2}} d x \leq C\|\nabla \Psi\|^{2-\beta_{2}}
$$

Then we have from (3.13) that

$\frac{d}{d t}\|\bar{p}\|^{2} \leq-\left(\frac{d_{2} \delta^{\beta_{1}+\beta_{2}}}{2^{1+\beta_{1}} C_{\mathrm{PS}}^{2-\beta_{2}}}-2 \varepsilon\right)\|\bar{p}\|^{2-\beta_{2}}+C\left(\delta^{2+\beta_{1}}+\|\nabla \Psi\|^{2-\beta_{2}}+\|\nabla \Psi\|^{2}+\varepsilon^{-\frac{1}{1-\beta_{2}}}\left\|\Psi_{t}\right\|^{\frac{2-\beta_{2}}{1-\beta_{2}}}\right)$.

Selecting $\varepsilon=d_{2} \delta^{\beta_{1}+\beta_{2}} /\left(2^{3+\beta_{1}} C_{\mathrm{PS}}^{2-\beta_{2}}\right)$ yields

$\frac{d}{d t}\|\bar{p}\|^{2} \leq-\frac{d_{2} \delta^{\beta_{1}+\beta_{2}}}{2^{2+\beta_{1}} C_{\mathrm{PS}}^{2-\beta_{2}}}\|\bar{p}\|^{2-\beta_{2}}+C\left(\delta^{2+\beta_{1}}+\|\nabla \Psi\|^{2-\beta_{2}}+\|\nabla \Psi\|^{2}+\delta^{-\frac{\beta_{1}+\beta_{2}}{1-\beta_{2}}}\left\|\Psi_{t}\right\|^{\frac{2-\beta_{2}}{1-\beta_{2}}}\right)$.

Denote $y(t)=\|\bar{p}(t)\|^{2}$. We rewrite (3.15) as

$$
\frac{d y}{d t} \leq-C_{0} \delta^{\beta_{1}+\beta_{2}} y^{\frac{2-\beta_{2}}{2}}+C\left(\delta^{2+\beta_{1}}+\|\nabla \Psi\|^{2-\beta_{2}}+\|\nabla \Psi\|^{2}+\delta^{-\frac{\beta_{1}+\beta_{2}}{1-\beta_{2}}}\left\|\Psi_{t}\right\|^{\frac{2-\beta_{2}}{1-\beta_{2}}}\right),
$$

where

$$
C_{0}=\frac{d_{2}}{2^{2+\beta_{1}} C_{\mathrm{PS}}^{2-\beta_{2}}}
$$

Select $\delta=1$. On the RHS of (3.16), we apply inequality (3.4) to have

$$
\|\nabla \Psi\|^{2-\beta_{2}} \leq 1+\|\nabla \Psi\|^{2} .
$$

Then

$$
\frac{d y}{d t} \leq-C_{0} y^{\frac{2-\beta_{2}}{2}}+C(1+f(t))
$$

Applying (3.7) and (3.8) in Lemma 3.2 to (3.18), we obtain (3.9) and (3.11), respectively. 
In case the boundary data is asymptotically small as $t \rightarrow \infty$, we prove in the next theorem that so is $\|\bar{p}(t)\|$.

Theorem 3.4. For any $\varepsilon>0$, there is $\delta_{0}>0$ such that if

$$
\limsup _{t \rightarrow \infty}\|\nabla \Psi(t)\| \leq \delta_{0} \quad \text { and } \quad \limsup _{t \rightarrow \infty}\left\|\Psi_{t}(t)\right\| \leq \delta_{0}
$$

then

$$
\limsup _{t \rightarrow \infty}\|\bar{p}(t)\| \leq \varepsilon
$$

Consequently, if

$$
\lim _{t \rightarrow \infty}\|\nabla \Psi(t)\|=\lim _{t \rightarrow \infty}\left\|\Psi_{t}(t)\right\|=0
$$

then

$$
\lim _{t \rightarrow \infty}\|\bar{p}(t)\|=0
$$

Proof. Let $\delta \in(0,1]$. Applying (3.8) in Lemma 3.2 to (3.16), and then using inequality (3.5) with $r=2 /\left(2-\beta_{2}\right)$ give

$$
\begin{aligned}
& \limsup _{t \rightarrow \infty}\|\bar{p}(t)\|^{2} \\
& \leq C\left\{\delta^{2-\beta_{2}}+\limsup _{t \rightarrow \infty}\left[\delta^{-\left(\beta_{1}+\beta_{2}\right)}\left(\|\nabla \Psi\|^{2-\beta_{2}}+\|\nabla \Psi\|^{2}\right)+\delta^{-\frac{\left(\beta_{1}+\beta_{2}\right)\left(2-\beta_{2}\right)}{1-\beta_{2}}}\left\|\Psi_{t}\right\|^{\frac{2-\beta_{2}}{1-\beta_{2}}}\right]\right\}^{\frac{2}{2-\beta_{2}}} \\
& \leq C\left\{\delta^{2}+\limsup _{t \rightarrow \infty}\left[\delta^{\frac{-2\left(\beta_{1}+\beta_{2}\right)}{2-\beta_{2}}}\left(\|\nabla \Psi\|^{2}+\|\nabla \Psi\|^{\frac{4}{2-\beta_{2}}}\right)+\delta^{-\frac{2\left(\beta_{1}+\beta_{2}\right)}{1-\beta_{2}}}\left\|\Psi_{t}\right\|^{\frac{2}{1-\beta_{2}}}\right]\right\} .
\end{aligned}
$$

Assume (3.19) with $0<\delta_{0} \leq 1$. It follows (3.23) that

$$
\limsup _{t \rightarrow \infty}\|\bar{p}(t)\|^{2} \leq C_{1}\left(\delta^{2}+3 \delta^{-\frac{2\left(\beta_{1}+\beta_{2}\right)}{1-\beta_{2}}} \delta_{0}^{2}\right)
$$

for some $C_{1}>0$. We choose $\delta$ sufficiently small so that $C_{1} \delta^{2} \leq \varepsilon^{2} / 2$, and then, with such $\delta$, choose $\delta_{0}$ to satisfy

$$
3 C_{1} \delta^{-\frac{2\left(\beta_{1}+\beta_{2}\right)}{1-\beta_{2}}} \delta_{0}^{2} \leq \varepsilon^{2} / 2 .
$$

Therefore, the desired estimate (3.20) follows (3.24).

In the case (3.21) is satisfied, we have (3.20) holds for any $\varepsilon>0$, which implies (3.22).

\subsection{Gradient estimates}

This subsection is focused on estimating the $L^{2-\beta_{2}}$-norm for $\nabla p(x, t)$. The following function $H(\xi)$ will be crucial in our gradient estimates.

Definition 3.5. Define for $\xi \geq 0$, the auxiliary function

$$
H(\xi)=\int_{0}^{\xi^{2}} K(\sqrt{s}) d s
$$

We compare $H(\xi)$ with $K(\xi) \xi^{2}$ and $\xi^{2-\beta_{2}}$ in the following lemma. 
Lemma 3.6. For any $\xi \geq 0$,

$$
\frac{d_{2}}{d_{3}} K(\xi) \xi^{2}-d_{2} K_{*}\left(\xi_{c}\right) \xi_{c}^{2} \leq H(\xi) \leq 2 K(\xi) \xi^{2}
$$

For any $\delta>0$ and $\xi \geq 0$,

$$
d_{2}\left(\frac{\delta}{1+\delta}\right)^{\beta_{1}+\beta_{2}}\left(\xi^{2-\beta_{2}}-\delta^{2-\beta_{2}}\right) \leq H(\xi) \leq 2 d_{3} \xi^{2-\beta_{2}} .
$$

Proof. By Corollary 2.3, the function $K(\xi) \xi$ is increasing, hence we have

$$
H(\xi)=2 \int_{0}^{\xi} K(s) s d s \leq 2 K(\xi) \xi \int_{0}^{\xi} 1 d s=2 K(\xi) \xi^{2} .
$$

This proves the second inequality of (3.26). Combining this with the second inequality in (2.5) for $m=2$ yields the second inequality in (3.27).

By Lemma 2.1,

$$
H(\xi) \geq d_{2} \int_{0}^{\xi^{2}} K_{*}(\sqrt{s}) d s .
$$

For $\xi>\xi_{c}$, using properties (P2) and (P3) of $K_{*}(\xi)$ in section 2, we have

$$
H(\xi) \geq d_{2} K_{*}(\xi) \int_{\xi_{c}^{2}}^{\xi^{2}} 1 d s=d_{2} K_{*}(\xi)\left(\xi^{2}-\xi_{c}^{2}\right) \geq d_{2}\left(K_{*}(\xi) \xi^{2}-K_{*}\left(\xi_{c}\right) \xi_{c}^{2}\right) .
$$

For $\xi \leq \xi_{c}$, according to Corollary 2.3 the function $\xi^{2} K(\xi)$ is increasing thus

$$
d_{2}\left(K_{*}(\xi) \xi^{2}-K_{*}\left(\xi_{c}\right) \xi_{c}^{2}\right) \leq 0 \leq H(\xi) .
$$

Combining the above two inequalities we have

$$
d_{2}\left(K_{*}(\xi) \xi^{2}-K_{*}\left(\xi_{c}\right) \xi_{c}^{2}\right) \leq H(\xi) .
$$

Applying (2.4) in Lemma 2.1 to compare $K_{*}(\xi)$ with $K(\xi)$ in (3.32) yields

$$
\frac{d_{2}}{d_{3}} K(\xi) \xi^{2}-d_{2} K_{*}\left(\xi_{c}\right) \xi_{c}^{2} \leq H(\xi)
$$

Hence, we obtain the first inequality of (3.26).

Next, we prove the first inequality of (3.27). Since it trivially holds true for all $\xi \leq \delta$, it suffices to consider $\xi>\delta$. From (3.28),

$$
H(\xi) \geq 2 \int_{\delta}^{\xi} K(s) s d s=2 \int_{\delta}^{\xi} K(s) s^{\beta_{2}} s^{1-\beta_{2}} d s .
$$

According to Corollary 2.3, the function $K(s) s^{\beta_{2}}$ is increasing thus

$$
H(\xi) \geq 2 K(\delta) \delta^{\beta_{2}} \int_{\delta}^{\xi} s^{1-\beta_{2}} d s=\frac{2}{2-\beta_{2}} K(\delta) \delta^{\beta_{2}}\left(\xi^{2-\beta_{2}}-\delta^{2-\beta_{2}}\right) \geq K(\delta) \delta^{\beta_{2}}\left(\xi^{2-\beta_{2}}-\delta^{2-\beta_{2}}\right),
$$

which, together with (2.4), proves the first inequality of (3.27). The proof is complete. 
Bounds for the gradient in terms of the initial and boundary data are obtained in the next theorem.

Theorem 3.7. For all $t \geq 0$,

$$
\begin{aligned}
\int_{U}|\nabla p(x, t)|^{2-\beta_{2}} d x \leq & C\left(1+\|\bar{p}(0)\|^{2}+e^{-\frac{t}{2}} \int_{U}|\nabla p(x, 0)|^{2-\beta_{2}} d x\right. \\
& \left.+[\operatorname{Envf}(t)]^{\frac{2}{2-\beta_{2}}}+\int_{0}^{t} e^{-\frac{1}{2}(t-\tau)}\left\|\nabla \Psi_{t}(\tau)\right\|^{2} d \tau\right) .
\end{aligned}
$$

Furthermore,

$$
\limsup _{t \rightarrow \infty} \int_{U}|\nabla p(x, t)|^{2-\beta_{2}} d x \leq C\left(1+\limsup _{t \rightarrow \infty} G_{1}(t)\right)
$$

where

$$
G_{1}(t)=G_{1}[\Psi](t) \stackrel{\text { def }}{=} f(t)^{\frac{2}{2-\beta_{2}}}+\left\|\nabla \Psi_{t}(t)\right\|^{2}
$$

Proof. Multiplying the first equation in the (3.2) by $\bar{p}_{t}$, integrating over the domain $U$, using integration by parts for the first integral on the RHS and by the fact that $\bar{p}_{t}=p_{t}-\Psi_{t}$; we have

$$
\begin{aligned}
\int_{U} \bar{p}_{t}^{2} d x & =-\int_{U} K(|\nabla p|) \nabla p \cdot \nabla \bar{p}_{t} d x-\int_{U} \bar{p}_{t} \Psi_{t} \\
& =-\int_{U} K(|\nabla p|) \nabla p \cdot \nabla p_{t} d x+\int_{U} K(|\nabla p|) \nabla p \cdot \nabla \Psi_{t} d x-\int_{U} \bar{p}_{t} \Psi_{t} d x .
\end{aligned}
$$

For the first integral on the RHS, using definition (3.25) of $H(\xi)$ we have

$$
\left\|\bar{p}_{t}\right\|^{2}+\frac{1}{2} \frac{d}{d t} \int_{U} \mathcal{H}(x, t) d x=\int_{U} K(|\nabla p|) \nabla p \cdot \nabla \Psi_{t} d x-\int_{U} \bar{p}_{t} \Psi_{t} d x
$$

where, for the sake of simplicity, we denoted

$$
\mathcal{H}(x, t)=H(|\nabla p(x, t)|) .
$$

Let

$$
\mathcal{E}(t)=\int_{U}|\bar{p}(x, t)|^{2} d x+\int_{U} \mathcal{H}(x, t) d x .
$$

Summing (3.12) and (3.36) gives

$$
\begin{aligned}
\left\|\bar{p}_{t}\right\|^{2}+\frac{1}{2} \frac{d}{d t} \mathcal{E}(t)= & -\int_{U} K(|\nabla p|)|\nabla p|^{2} d x+\int_{U} K(|\nabla p|) \nabla p \cdot \nabla\left(\Psi+\Psi_{t}\right) d x \\
& -\int_{U} \Psi_{t}\left(\bar{p}+\bar{p}_{t}\right) d x=I_{1}+I_{2}+I_{3} .
\end{aligned}
$$

It follows from $(3.26)$ that

$$
I_{1} \leq-\frac{1}{2} \int_{U} \mathcal{H}(x, t) d x
$$

Let $\varepsilon>0$. Applying Cauchy's inequality for $I_{2}$, and using the fact (2.18) that $K(\cdot)$ bounded

$$
\left|I_{2}\right| \leq \varepsilon \int_{U} K(|\nabla p|)|\nabla p|^{2} d x+C \varepsilon^{-1}\left(\|\nabla \Psi\|^{2}+\left\|\nabla \Psi_{t}\right\|^{2}\right) .
$$


Again using (3.26),

$$
\left|I_{2}\right| \leq \varepsilon \int_{U}\left(C_{1} \mathcal{H}(x, t)+C_{2}\right) d x+C \varepsilon^{-1}\left(\|\nabla \Psi\|^{2}+\left\|\nabla \Psi_{t}\right\|^{2}\right),
$$

where $C_{1}=d_{3} / d_{2}$ and $C_{2}=d_{3} K_{*}\left(\xi_{c}\right) \xi_{c}^{2}$.

For $I_{3}$, applying Cauchy's inequality gives

$$
\left|I_{3}\right| \leq \frac{1}{2}\left(\|\bar{p}\|^{2}+\left\|\bar{p}_{t}\right\|^{2}\right)+\left\|\Psi_{t}\right\|^{2} .
$$

Combining (3.38)-(3.41), we obtain

$\frac{d}{d t} \mathcal{E}(t)+\left\|\bar{p}_{t}\right\|^{2} \leq-\left(1-2 \varepsilon C_{1}\right) \int_{U} \mathcal{H}(x, t) d x+\|\bar{p}\|^{2}+2 \varepsilon C_{2}|U|+C \varepsilon^{-1}\left(\|\nabla \Psi\|^{2}+\left\|\nabla \Psi_{t}\right\|^{2}\right)+2\left\|\Psi_{t}\right\|^{2}$.

Selecting $\varepsilon=\delta /\left(4 C_{1}\right)$ in (3.42) with $\delta \in(0,1]$ and using (3.37), we find that

$$
\begin{aligned}
\frac{d}{d t} \mathcal{E}(t)+\left\|\bar{p}_{t}\right\|^{2} & \leq-\frac{1}{2} \int_{U} \mathcal{H}(x, t) d x+\|\bar{p}\|^{2}+C \delta+C \delta^{-1}\left(\|\nabla \Psi\|^{2}+\left\|\nabla \Psi_{t}\right\|^{2}\right)+2\left\|\Psi_{t}\right\|^{2} \\
& \leq-\frac{1}{2} \mathcal{E}(t)+\frac{3}{2}\|\bar{p}\|^{2}+C \delta+C \delta^{-1}\left(\|\nabla \Psi\|^{2}+\left\|\nabla \Psi_{t}\right\|^{2}\right)+2\left\|\Psi_{t}\right\|^{2} .
\end{aligned}
$$

Letting $\delta=1$,

$$
\frac{d}{d t} \mathcal{E}(t)+\left\|\bar{p}_{t}\right\|^{2} \leq-\frac{1}{2} \mathcal{E}(t)+\frac{3}{2}\|\bar{p}\|^{2}+C\left(1+\|\nabla \Psi\|^{2}+\left\|\nabla \Psi_{t}\right\|^{2}+\left\|\Psi_{t}\right\|^{2}\right) .
$$

Thanks to estimate (3.9) of $\|\bar{p}(t)\|^{2}$ and by using (3.4) to bound

$$
\left\|\Psi_{t}\right\|^{2} \leq 1+\left\|\Psi_{t}\right\|^{\frac{2-\beta_{2}}{1-\beta_{2}}}
$$

we obtain

$$
\frac{d}{d t} \mathcal{E}(t) \leq-\frac{1}{2} \mathcal{E}(t)+\frac{3}{2}\|\bar{p}(0)\|^{2}+C+C\left([\operatorname{Env} f(t)]^{\frac{2}{2-\beta_{2}}}+\left\|\nabla \Psi_{t}(t)\right\|^{2}\right) .
$$

It follows from Gronwall's inequality that

$$
\mathcal{E}(t) \leq e^{-\frac{t}{2}} \mathcal{E}(0)+3\|\bar{p}(0)\|^{2}+C+C \int_{0}^{t} e^{-\frac{1}{2}(t-\tau)}\left([\operatorname{Envf}(\tau)]^{\frac{2}{2-\beta_{2}}}+\left\|\nabla \Psi_{t}(\tau)\right\|^{2}\right) d \tau,
$$

which, by monotonicity of function $\operatorname{Env} f(t)$ and definition of $\mathcal{E}(t)$, leads to

$$
\begin{aligned}
\int_{U} \mathcal{H}(x, t) d x \leq 4\|\bar{p}(0)\|^{2}+e^{-\frac{t}{2}} \int_{U} \mathcal{H}(x, 0) d x & \\
& +C+C[\operatorname{Envf}(t)]^{\frac{2}{2-\beta_{2}}}+C \int_{0}^{t} e^{-\frac{1}{2}(t-\tau)}\left\|\nabla \Psi_{t}(\tau)\right\|^{2} d \tau .
\end{aligned}
$$

We bound $\mathcal{H}(x, t)$ from below by the first inequality in (3.27) with $\delta=1$, and bound $\mathcal{H}(x, 0)$ from above by the second inequality of (3.27). It results in

$$
\begin{aligned}
C_{3} \int_{U}|\nabla p(x, t)|^{2-\beta_{2}} d x-C_{4} \leq 4\|\bar{p}(0)\|^{2} & +2 d_{3} e^{-\frac{t}{2}} \int_{U}|\nabla p(x, 0)|^{2-\beta_{2}} d x \\
& +C+C[\operatorname{Envf(t)}]^{\frac{2}{2-\beta_{2}}}+C \int_{0}^{t} e^{-\frac{1}{2}(t-\tau)}\left\|\nabla \Psi_{t}(\tau)\right\|^{2} d \tau
\end{aligned}
$$


for constants $C_{3}=d_{2} / 2^{\beta_{1}+\beta_{2}}$ and $C_{4}=C_{3}|U|$, and estimate (3.34) follows.

Neglecting $\left\|\bar{p}_{t}\right\|^{2}$ on the LHS from (3.44), we have

$$
\frac{d}{d t} \mathcal{E}(t) \leq-\frac{1}{2} \mathcal{E}(t)+\frac{3}{2}\|\bar{p}\|^{2}+C\left(1+\|\nabla \Psi\|^{2}+\left\|\nabla \Psi_{t}\right\|^{2}+\left\|\Psi_{t}\right\|^{2}\right) .
$$

Applying (3.8) in Lemma 3.2 to (3.46), we have

$$
\limsup _{t \rightarrow \infty} \mathcal{E}(t) \leq 3 \limsup _{t \rightarrow \infty}\|\bar{p}\|^{2}+C \limsup _{t \rightarrow \infty}\left(1+\|\nabla \Psi\|^{2}+\left\|\nabla \Psi_{t}\right\|^{2}+\left\|\Psi_{t}\right\|^{2}\right) .
$$

Combining this with (3.11) yields

$$
\limsup _{t \rightarrow \infty} \int_{U} \mathcal{H}(x, t) d x \leq C\left(1+\limsup _{t \rightarrow \infty} G_{1}(t)\right) .
$$

Again, by using the first inequality in (3.27) with $\delta=1$ to bound $\mathcal{H}(x, t)$ from below in terms of $|\nabla p(x, t)|^{2-\beta_{2}}$, we obtain estimate (3.35) from (3.47).

Below is a counterpart of Theorem 3.4, but for the gradient instead.

Theorem 3.8. For any $\varepsilon>0$, there is $\delta_{0}>0$ such that if

$$
\limsup _{t \rightarrow \infty}\left(\|\nabla \Psi(t)\|+\left\|\Psi_{t}(t)\right\|+\left\|\nabla \Psi_{t}(t)\right\|\right) \leq \delta_{0}
$$

then

$$
\limsup _{t \rightarrow \infty} \int_{U}|\nabla p(x, t)|^{2-\beta_{2}} d x \leq \varepsilon
$$

Consequently, if

$$
\lim _{t \rightarrow \infty}\|\nabla \Psi(t)\|=\lim _{t \rightarrow \infty}\left\|\Psi_{t}(t)\right\|=\lim _{t \rightarrow \infty}\left\|\nabla \Psi_{t}(t)\right\|=0
$$

then

$$
\lim _{t \rightarrow \infty} \int_{U}|\nabla p(x, t)|^{2-\beta_{2}} d x=0 .
$$

Proof. First, we estimate the limit superior of $\int_{U} \mathcal{H}(x, t) d x$ as $t \rightarrow \infty$. Let $\delta \in(0,1]$. Applying (3.8) in Lemma 3.2 to (3.43), we have

$$
\limsup _{t \rightarrow \infty} \mathcal{E}(t) \leq C \limsup _{t \rightarrow \infty}\|\bar{p}\|^{2}+C\left\{\delta^{-1} \limsup _{t \rightarrow \infty}\left(\|\nabla \Psi\|^{2}+\left\|\nabla \Psi_{t}\right\|^{2}\right)+\limsup _{t \rightarrow \infty}\left\|\Psi_{t}\right\|^{2}+\delta\right\} .
$$

It follows from (3.52) and (3.23) that

$$
\begin{aligned}
& \limsup _{t \rightarrow \infty} \int_{U} \mathcal{H}(x, t) d x \\
& \leq C\left\{\delta^{2}+\delta^{-\frac{2\left(\beta_{1}+\beta_{2}\right)}{2-\beta_{2}}}\left(\limsup _{t \rightarrow \infty}\|\nabla \Psi\|^{2}+\limsup _{t \rightarrow \infty}\|\nabla \Psi\|^{\frac{4}{2-\beta_{2}}}\right)+\delta^{-\frac{2\left(\beta_{1}+\beta_{2}\right)}{1-\beta_{2}}} \limsup _{t \rightarrow \infty}\left\|\Psi_{t}\right\|^{\frac{2}{1-\beta_{2}}}\right\} \\
& \quad+C\left\{\delta^{-1} \limsup _{t \rightarrow \infty}\left(\|\nabla \Psi\|^{2}+\left\|\nabla \Psi_{t}\right\|^{2}\right)+\limsup _{t \rightarrow \infty}\left\|\Psi_{t}\right\|^{2}+\delta\right\} .
\end{aligned}
$$

Thanks to the fact $\delta \leq 1$, it follows that

$$
\begin{aligned}
& \limsup _{t \rightarrow \infty} \int_{U} \mathcal{H}(x, t) d x \leq C\left\{\delta+\delta^{-\kappa} \limsup _{t \rightarrow \infty}\left(\|\nabla \Psi(t)\|^{2}+\|\nabla \Psi(t)\|^{\frac{4}{2-\beta_{2}}}\right.\right. \\
&\left.\left.+\left\|\Psi_{t}(t)\right\|^{\frac{2}{1-\beta_{2}}}+\left\|\Psi_{t}(t)\right\|^{2}+\left\|\nabla \Psi_{t}(t)\right\|^{2}\right)\right\},
\end{aligned}
$$


where $\kappa=\max \left\{\frac{2\left(\beta_{1}+\beta_{2}\right)}{1-\beta_{2}}, 1\right\}$.

Let $\delta_{1}$ be any number in $(0,1]$. Applying the first inequality of (3.27) with $\delta=\delta_{1}$ gives

$$
\mathcal{H}(x, t) \geq \frac{d_{2} \delta_{1}^{\beta_{1}+\beta_{2}}}{2^{\beta_{1}+\beta_{2}}}|\nabla p(x, t)|^{2-\beta_{2}}-d_{2} \delta_{1}^{2+\beta_{1}} .
$$

Combining this with (3.53) yields

$$
\begin{aligned}
\limsup _{t \rightarrow \infty} \int_{U}|\nabla p(x, t)|^{2-\beta_{2}} d x \leq & C \delta_{1}^{-\left(\beta_{1}+\beta_{2}\right)} \limsup _{t \rightarrow \infty} \int_{U} \mathcal{H}(x, t) d x+C \delta_{1}^{2-\beta_{2}} \\
\leq & C \delta_{1}^{-\left(\beta_{1}+\beta_{2}\right)}\left\{\delta+\delta^{-\kappa} \limsup _{t \rightarrow \infty}\left(\|\nabla \Psi\|^{2}+\|\nabla \Psi\|^{\frac{4}{2-\beta_{2}}}\right.\right. \\
& \left.\left.+\left\|\Psi_{t}\right\|^{\frac{2}{1-\beta_{2}}}+\left\|\Psi_{t}\right\|^{2}+\left\|\nabla \Psi_{t}\right\|^{2}\right)\right\}+C \delta_{1}^{2-\beta_{2}} .
\end{aligned}
$$

Assume (3.48) with $\delta_{0} \in(0,1]$, then (3.55) yields

$$
\begin{aligned}
\limsup _{t \rightarrow \infty} \int_{U}|\nabla p|^{2-\beta_{2}} d x & \leq C_{5} \delta_{1}^{-\left(\beta_{1}+\beta_{2}\right)}\left\{\delta+\delta^{-\kappa}\left(\delta_{0}^{2}+\delta_{0}^{\frac{4}{2-\beta_{2}}}+\delta_{0}^{\frac{2}{1-\beta_{2}}}\right)\right\}+C_{5} \delta_{1}^{2-\beta_{2}} \\
& \leq C_{5} \delta_{1}^{-\left(\beta_{1}+\beta_{2}\right)}\left(\delta+3 \delta^{-\kappa} \delta_{0}^{2}\right)+C_{5} \delta_{1}^{2-\beta_{2}}
\end{aligned}
$$

for some $C_{5}>0$ is independent of $\delta, \delta_{0}$, and $\delta_{1}$.

First we choose $\delta_{1}$ sufficiently small satisfying $C_{5} \delta_{1}^{2-\beta_{2}} \leq \varepsilon / 3$. With this $\delta_{1}$, choose $\delta \in(0,1]$ such that $C_{5} \delta_{1}^{-\left(\beta_{1}+\beta_{2}\right)} \delta \leq \varepsilon / 3$. Next, we choose $\delta_{0}>0$ much smaller than $\delta_{1}, \delta$ that satisfies

$$
C_{5} \delta_{1}^{-\left(\beta_{1}+\beta_{2}\right)} \delta^{-\kappa} \delta_{0}^{2} \leq \varepsilon / 9 .
$$

Then (3.49) follows (3.56). Finally, under condition (3.50), the estimate (3.49) holds for all $\varepsilon>0$, which proves (3.51).

When time $t$ is large, we improve the estimates in Theorem 3.7 by deriving uniform Gronwalltype inequalities.

Theorem 3.9. If $t \geq 1$ then

$$
\int_{t-\frac{1}{2}}^{t}\left\|\bar{p}_{t}(\tau)\right\|^{2} d \tau+\int_{U}|\nabla p(x, t)|^{2-\beta_{2}} d x \leq C\left(1+\|\bar{p}(t-1)\|^{2}+\int_{t-1}^{t}\left(f(\tau)+\left\|\nabla \Psi_{t}(\tau)\right\|^{2}\right) d \tau\right),
$$

and, consequently,

$$
\int_{U}|\nabla p(x, t)|^{2-\beta_{2}} d x \leq C\left(1+\|\bar{p}(0)\|^{2}+(\operatorname{Envf}(t))^{\frac{2}{2-\beta_{2}}}+\int_{t-1}^{t}\left\|\nabla \Psi_{t}(\tau)\right\|^{2} d \tau\right) .
$$

Proof. On the right-hand side of (3.13), we use (3.14) again but this time to bound $\|\bar{p}\|$ in terms of $\int_{U}|\nabla \bar{p}|^{2-\beta_{2}} d x$. Then, with the same choice of $\varepsilon$, we have instead of (3.15)

$$
\frac{d}{d t}\|\bar{p}\|^{2} \leq-\frac{d_{2} \delta^{\beta_{1}+\beta_{2}}}{2^{2+\beta_{1}}} \int_{U}|\nabla \bar{p}|^{2-\beta_{2}} d x+C\left(\delta^{2+\beta_{1}}+\|\nabla \Psi\|^{2-\beta_{2}}+\|\nabla \Psi\|^{2}+\delta^{-\frac{\beta_{1}+\beta_{2}}{1-\beta_{2}}}\left\|\Psi_{t}\right\|^{\frac{2-\beta_{2}}{1-\beta_{2}}}\right) .
$$

Integrating (3.59) in time from $t-1$ to $t$, we have

$$
\begin{aligned}
\|\bar{p}(t)\|^{2}+C_{1} \delta^{\beta_{1}+\beta_{2}} \int_{t-1}^{t} \int_{U}|\nabla \bar{p}|^{2-\beta_{2}} d x d \tau \leq\|\bar{p}(t-1)\|^{2}+C \delta^{2+\beta_{1}} & \\
& +C \int_{t-1}^{t}\left(\|\nabla \Psi\|^{2-\beta_{2}}+\|\nabla \Psi\|^{2}+\delta^{-\frac{\beta_{1}+\beta_{2}}{1-\beta_{2}}}\left\|\Psi_{t}\right\|^{\frac{2-\beta_{2}}{1-\beta_{2}}}\right) d \tau
\end{aligned}
$$


where $C_{1}>0$ is independent of $\delta$.

Let $\varepsilon \in(0,1]$. Applying Cauchy's inequality to integrals on the RHS of (3.36), we have the following:

$$
\left\|\bar{p}_{t}\right\|^{2}+\frac{1}{2} \frac{d}{d t} \int_{U} \mathcal{H}(x, t) d x \leq \varepsilon \int_{U} K(|\nabla p|)|\nabla p|^{2} d x+\frac{C}{\varepsilon} \int_{U} K(|\nabla p|)\left|\nabla \Psi_{t}\right|^{2} d x+\frac{1}{2}\left\|\bar{p}_{t}\right\|^{2}+\frac{1}{2}\left\|\Psi_{t}\right\|^{2} .
$$

Then using (2.18) for the second integral on the RHS of (3.61), we have

$$
\left\|\bar{p}_{t}\right\|^{2}+\frac{d}{d t} \int_{U} \mathcal{H}(x, t) d x \leq 2 \varepsilon \int_{U} K(|\nabla p|)|\nabla p|^{2} d x+C\left(\varepsilon^{-1}\left\|\nabla \Psi_{t}\right\|^{2}+\left\|\Psi_{t}\right\|^{2}\right) .
$$

By virtue of (3.26), we find from (3.62) that

$$
\left\|\bar{p}_{t}\right\|^{2}+\frac{d}{d t} \int_{U} \mathcal{H}(x, t) d x \leq C \varepsilon \int_{U} \mathcal{H}(x, t) d x+C\left(\varepsilon+\varepsilon^{-1}\left\|\nabla \Psi_{t}\right\|^{2}+\left\|\Psi_{t}\right\|^{2}\right) .
$$

Integrating (3.63) in time from $s$ to $t$ where $s \in[t-1, t]$, we have

$$
\begin{aligned}
& \int_{s}^{t}\left\|\bar{p}_{t}\right\|^{2} d \tau+\int_{U} \mathcal{H}(x, t) d x \\
& \quad \leq \int_{U} \mathcal{H}(x, s) d x+C \varepsilon \int_{s}^{t} \int_{U} \mathcal{H}(x, \tau) d x d \tau+C \int_{s}^{t}\left(\varepsilon+\varepsilon^{-1}\left\|\nabla \Psi_{t}\right\|^{2}+\left\|\Psi_{t}\right\|^{2}\right) d \tau \\
& \quad \leq \int_{U} \mathcal{H}(x, s) d x+C \int_{t-1}^{t} \int_{U} \mathcal{H}(x, t) d x d \tau+C \varepsilon+C \int_{t-1}^{t}\left(\varepsilon^{-1}\left\|\nabla \Psi_{t}\right\|^{2}+\left\|\Psi_{t}\right\|^{2}\right) d \tau
\end{aligned}
$$

Integrating (3.64) in $s$ from $t-1$ to $t$ shows that

$$
\int_{t-1}^{t} \int_{s}^{t}\left\|\bar{p}_{t}\right\|^{2} d \tau d s+\int_{U} \mathcal{H}(x, t) d x \leq C\left\{\int_{t-1}^{t} \int_{U} \mathcal{H}(x, \tau) d x d \tau+\varepsilon+\int_{t-1}^{t}\left(\varepsilon^{-1}\left\|\nabla \Psi_{t}\right\|^{2}+\left\|\Psi_{t}\right\|^{2}\right) d \tau\right\} .
$$

Estimating first term of (3.65) by

$$
\int_{t-1}^{t} \int_{s}^{t}\left\|\bar{p}_{t}\right\|^{2} d \tau d s \geq \int_{t-1}^{t-\frac{1}{2}} \int_{t-\frac{1}{2}}^{t}\left\|\bar{p}_{t}\right\|^{2} d \tau d s \geq \frac{1}{2} \int_{t-\frac{1}{2}}^{t}\left\|\bar{p}_{t}\right\|^{2} d \tau
$$

we get

$$
\frac{1}{2} \int_{t-\frac{1}{2}}^{t}\left\|\bar{p}_{t}\right\|^{2} d \tau+\int_{U} \mathcal{H}(x, t) d x \leq C\left\{\int_{t-1}^{t} \int_{U} \mathcal{H}(x, \tau) d x d \tau+\varepsilon+\int_{t-1}^{t}\left(\varepsilon^{-1}\left\|\nabla \Psi_{t}\right\|^{2}+\left\|\Psi_{t}\right\|^{2}\right) d \tau\right\} .
$$

Estimating the double integral on the RHS of (3.66) by combining the second inequality of (3.27) with (3.60), we obtain

$$
\begin{aligned}
& \frac{1}{2} \int_{t-\frac{1}{2}}^{t}\left\|\bar{p}_{t}(\tau)\right\|^{2} d \tau+\int_{U} \mathcal{H}(x, t) d x \\
& \leq C\left\{\delta^{-\left(\beta_{1}+\beta_{2}\right)}\|\bar{p}(t-1)\|^{2}+\delta^{2-\beta_{2}}+\delta^{-\left(\beta_{1}+\beta_{2}\right)} \int_{t-1}^{t}\left(\|\nabla \Psi\|^{2-\beta_{2}}+\|\nabla \Psi\|^{2}+\delta^{-\frac{\beta_{1}+\beta_{2}}{1-\beta_{2}}}\left\|\Psi_{t}\right\|^{\frac{2-\beta_{2}}{1-\beta_{2}}}\right) d \tau\right. \\
& \left.\quad+\varepsilon+\int_{t-1}^{t}\left(\varepsilon^{-1}\left\|\nabla \Psi_{t}\right\|^{2}+\left\|\Psi_{t}\right\|^{2}\right) d \tau\right\} . \quad(3.67)
\end{aligned}
$$


In (3.67), choosing $\varepsilon=\delta=1$, using (3.17) and (3.45) give

$$
\begin{aligned}
\int_{t-1 / 2}^{t}\left\|\bar{p}_{t}\right\|^{2} d \tau+\int_{U} \mathcal{H}(x, t) d x & \leq C\left(1+\|\bar{p}(t-1)\|^{2}+\int_{t-1}^{t}\left(\|\nabla \Psi\|^{2}+\left\|\nabla \Psi_{t}\right\|^{2}+\left\|\Psi_{t}\right\|^{\frac{2-\beta_{2}}{1-\beta_{2}}}\right) d \tau\right) \\
& =C\left(1+\|\bar{p}(t-1)\|^{2}+\int_{t-1}^{t}\left(f(\tau)+\left\|\nabla \Psi_{t}(\tau)\right\|^{2}\right) d \tau\right) .
\end{aligned}
$$

Combining this with the first inequality in (3.27) with $\delta=1$ yields (3.57).

Combining (3.57) with (3.9), we obtain (3.58).

As for $t \rightarrow \infty$, we have the following alternative results.

Corollary 3.10. Ones have

$$
\limsup _{t \rightarrow \infty} \int_{U}|\nabla p(x, t)|^{2-\beta_{2}} d x \leq C\left(1+\limsup _{t \rightarrow \infty} f(t)^{\frac{2}{2-\beta_{2}}}+\limsup _{t \rightarrow \infty} \int_{t-1}^{t}\left\|\nabla \Psi_{t}(\tau)\right\|^{2} d \tau\right) .
$$

Moreover, if

$$
\lim _{t \rightarrow \infty}\|\nabla \Psi(t)\|=\lim _{t \rightarrow \infty}\left\|\Psi_{t}(t)\right\|=\lim _{t \rightarrow \infty} \int_{t-1}^{t}\left\|\nabla \Psi_{t}(\tau)\right\|^{2} d \tau=0
$$

then

$$
\lim _{t \rightarrow \infty} \int_{U}|\nabla p(x, t)|^{2-\beta_{2}} d x=0 .
$$

Proof. Combining (3.11) and the limit superior of (3.57), we have

$$
\begin{aligned}
& \limsup _{t \rightarrow \infty} \int_{U}|\nabla p(x, t)|^{2-\beta_{2}} d x \\
& \leq C\left(1+\limsup _{t \rightarrow \infty} f(t)^{\frac{2}{2-\beta_{2}}}+\limsup _{t \rightarrow \infty} \int_{t-1}^{t}\left(\|\nabla \Psi\|^{2}+\left\|\nabla \Psi_{t}\right\|^{2}+\left\|\Psi_{t}\right\|^{\frac{2-\beta_{2}}{1-\beta_{2}}}\right) d \tau\right),
\end{aligned}
$$

which implies (3.68).

Let $\delta_{1} \in(0,1]$. On the left-hand side of (3.67), we neglect the time derivative term and apply (3.54) to bound $\mathcal{H}(x, t)$ from below in terms of $|\nabla p(x, t)|^{2-\beta_{2}}$. It yields

$$
\begin{array}{r}
\delta_{1}^{\beta_{1}+\beta_{2}} \int_{U}|\nabla p(x, t)|^{2-\beta_{2}} d x \leq C\left\{\delta_{1}^{2+\beta_{1}}+\delta^{2-\beta_{2}}+\delta^{-\left(\beta_{1}+\beta_{2}\right)}\|\bar{p}(t-1)\|^{2}\right. \\
+\delta^{-\left(\beta_{1}+\beta_{2}\right)} \int_{t-1}^{t}\left(\|\nabla \Psi\|^{2-\beta_{2}}+\|\nabla \Psi\|^{2}+\delta^{-\frac{\beta_{1}+\beta_{2}}{1-\beta_{2}}}\left\|\Psi_{t}\right\|^{\frac{2-\beta_{2}}{1-\beta_{2}}}\right) d \tau \\
\left.+\varepsilon+\int_{t-1}^{t}\left(\varepsilon^{-1}\left\|\nabla \Psi_{t}\right\|^{2}+\left\|\Psi_{t}\right\|^{2}\right) d \tau\right\}
\end{array}
$$

Under condition (3.69), we have from Theorem 3.4 that $\lim _{t \rightarrow \infty}\|\bar{p}(t-1)\|=0$. Passing $t \rightarrow \infty$ in (3.71) gives

$$
\begin{aligned}
\limsup _{t \rightarrow \infty} \int_{U}|\nabla p(x, t)|^{2-\beta_{2}} d x & \leq C \delta_{1}^{-\left(\beta_{1}+\beta_{2}\right)}\left(\delta_{1}^{2+\beta_{1}}+\delta^{2-\beta_{2}}+\varepsilon\right) \\
& =C \delta_{1}^{2-\beta_{2}}+C \delta_{1}^{-\left(\beta_{1}+\beta_{2}\right)}\left(\delta^{2-\beta_{2}}+\varepsilon\right) .
\end{aligned}
$$

Letting $\varepsilon \rightarrow 0, \delta \rightarrow 0$, and then $\delta_{1} \rightarrow 0$, we obtain (3.70). 
Remark 3.11. (a) Comparing with (3.34), the inequality (3.58) explicitly shows the independence on the initial norm $\|\nabla p(0)\|_{L^{2-\beta_{2}}}$. Also, the term $\int_{t-1}^{t}\left\|\nabla \Psi_{t}(\tau)\right\|^{2} d \tau$ explicitly shows that the dependence on the second derivative $\nabla \Psi_{t}$ of the boundary data is not accumulative in time on the whole interval $(0, t)$.

(b) Since

$$
\limsup _{t \rightarrow \infty} \int_{t-1}^{t}\left\|\nabla \Psi_{t}(\tau)\right\|^{2} d \tau \leq \limsup _{t \rightarrow \infty}\left\|\nabla \Psi_{t}(t)\right\|^{2},
$$

the results (3.68) - (3.70) in Corollary 3.10 improve (3.35) in Theorem 3.7 and (3.48) -(3.51) in Theorem 3.8 .

\section{Continuous dependence on the initial and boundary data}

In this section, we establish the continuous dependence of the solution of problem (3.1) on the initial and boundary data. We consider $K(\xi)=\bar{K}(\xi), K_{I}(\xi), \hat{K}(\xi), K_{M}(\xi)$.

Let $p_{1}(x, t)$ and $p_{2}(x, t)$ be two solutions of (3.1) with boundary data $\psi_{1}(x, t)$ and $\psi_{2}(x, t)$, respectively. For $i=1,2$, let $\Psi_{i}(x, t)$ be an extension of $\psi_{i}(x, t)$ to $\bar{U} \times[0, \infty)$, and $\bar{p}_{i}=p_{i}-\Psi_{i}$. Denote

$$
\Phi=\Psi_{1}-\Psi_{2} \quad \text { and } \quad \bar{P}=\bar{p}_{1}-\bar{p}_{2}=p_{1}-p_{2}-\Phi
$$

Then

$$
\begin{aligned}
\frac{\partial \bar{P}}{\partial t} & =\nabla \cdot\left(K\left(\left|\nabla p_{1}\right|\right) \nabla p_{1}\right)-\nabla \cdot\left(K\left(\left|\nabla p_{2}\right|\right) \nabla p_{2}\right)-\Phi_{t} \quad \text { on } U \times(0, \infty), \\
\bar{P} & =0 \quad \text { on } \Gamma \times(0, \infty) .
\end{aligned}
$$

Let

$$
\Lambda(t)=1+\left\|\nabla p_{1}(t)\right\|_{L^{2-\beta_{2}}}+\left\|\nabla p_{2}(t)\right\|_{L^{2-\beta_{2}}} .
$$

For the difference between two boundary data, we define

$$
D(t)=\left\|\Phi_{t}(t)\right\|+\|\nabla \Phi(t)\|_{L^{2-\beta_{2}}}+\|\nabla \Phi(t)\|_{L^{2+\beta_{1}}}^{2+\beta_{1}} .
$$

First, we obtain the estimates for $\|\bar{P}(t)\|$ in terms of $D(t)$ and individual solutions $p_{1}(x, t)$, $p_{2}(x, t)$.

Proposition 4.1. If $t \geq 0$ then

$$
\begin{aligned}
& \|\bar{P}(t)\|^{2} \leq\|\bar{P}(0)\|^{2}+C\left\{\operatorname{Env}\left[\Lambda(t)^{\beta_{1}+\beta_{2}}\left(\Lambda(t)^{1-\beta_{2}}+\left\|\bar{p}_{1}(t)\right\|+\left\|\bar{p}_{2}(t)\right\|\right) D(t)\right]\right\}^{\frac{2}{2+\beta_{1}}} . \\
& \text { If } \int_{0}^{\infty} \Lambda(t)^{-\left(\beta_{1}+\beta_{2}\right)} d t=\infty, \text { then } \\
& \limsup _{t \rightarrow \infty}\|\bar{P}(t)\|^{2} \leq C \limsup _{t \rightarrow \infty}\left\{\Lambda(t)^{\beta_{1}+\beta_{2}}\left(\Lambda(t)^{1-\beta_{2}}+\left\|\bar{p}_{1}(t)\right\|+\left\|\bar{p}_{2}(t)\right\|\right) D(t)\right\}^{\frac{2}{2+\beta_{1}}} .
\end{aligned}
$$

Proof. First, we find a differential inequality for $\|\bar{P}(t)\|^{2}$. We define

$$
\omega(x, t)=1+\left|\nabla p_{2}(x, t)\right|+\left|\nabla p_{1}(x, t)\right| .
$$


Multiplying (4.1) by $\bar{P}$, integrating the resulting equation over $U$, and using integration by parts, we obtain

$$
\frac{1}{2} \frac{d}{d t} \int_{U} \bar{P}^{2} d x=-\int_{U}\left[K\left(\left|\nabla p_{1}\right|\right) \nabla p_{1}-K\left(\left|\nabla p_{2}\right|\right) \nabla p_{2}\right] \cdot \nabla \bar{P} d x-\int_{U} \Phi_{t} \bar{P} d x
$$

thus,

$$
\begin{aligned}
\frac{1}{2} \frac{d}{d t} \int_{U} \bar{P}^{2} d x= & -\int_{U}\left[K\left(\left|\nabla p_{1}\right|\right) \nabla p_{1}-K\left(\left|\nabla p_{2}\right|\right) \nabla p_{2}\right] \cdot \nabla\left(p_{1}-p_{2}\right) d x \\
& +\int_{U}\left[K\left(\left|\nabla p_{1}\right|\right) \nabla p_{1}-K\left(\left|\nabla p_{2}\right|\right) \nabla p_{2}\right] \cdot \nabla \Phi d x-\int_{U} \Phi_{t} \bar{P} d x
\end{aligned}
$$

Using the monotonicity in Lemma 2.4 for the first integral on the RHS, and property (2.5) with $m=1$ for the second integral, we have

$$
\begin{aligned}
\frac{1}{2} \frac{d}{d t} \int_{U} \bar{P}^{2} d x \leq & -d_{5} \int_{U} \frac{\left|\nabla\left(p_{1}-p_{2}\right)\right|^{2+\beta_{1}}}{\omega^{\beta_{1}+\beta_{2}}} d x+d_{3} \int_{U}\left(\left|\nabla p_{1}\right|^{1-\beta_{2}}+\left|\nabla p_{2}\right|^{1-\beta_{2}}\right)|\nabla \Phi| d x \\
& +\int_{U}\left|\Phi_{t}\right|\left(\left|\bar{p}_{1}\right|+\left|\bar{p}_{2}\right|\right) d x \stackrel{\text { def }}{=}-J_{1}+J_{2}+J_{3} .
\end{aligned}
$$

By (3.6),

$$
\begin{aligned}
-J_{1} & \leq-C_{1} \int_{U} \frac{|\nabla \bar{P}|^{2+\beta_{1}}}{\omega^{\beta_{1}+\beta_{2}}} d x+C \int_{U} \frac{|\nabla \Phi|^{2+\beta_{1}}}{\omega^{\beta_{1}+\beta_{2}}} d x \\
& \leq-C_{1} \int_{U} \frac{|\nabla \bar{P}|^{2+\beta_{1}}}{\omega^{\beta_{1}+\beta_{2}}} d x+C \int_{U}|\nabla \Phi|^{2+\beta_{1}} d x,
\end{aligned}
$$

where $C_{1}=d_{5} / 2^{1+\beta_{1}}$. Using Hölder's inequality for $J_{2}$ and $J_{3}$ terms in (4.5), we find that

$$
\begin{gathered}
J_{2} \leq C\left(\left\|\nabla p_{1}\right\|_{L^{2-\beta_{2}}}^{1-\beta_{2}}+\left\|\nabla p_{2}\right\|_{L^{2-\beta_{2}}}^{1-\beta_{2}}\right)\|\nabla \Phi\|_{L^{2-\beta_{2}}} . \\
J_{3} \leq C\left(\left\|\bar{p}_{1}\right\|+\left\|\bar{p}_{2}\right\|\right)\left\|\Phi_{t}\right\| .
\end{gathered}
$$

Utilizing estimates (4.6)-(4.8) in (4.5), we obtain

$\frac{d}{d t}\|\bar{P}\|^{2} \leq-2 C_{1} \int_{U} \frac{|\nabla \bar{P}|^{2+\beta_{1}}}{\omega^{\beta_{1}+\beta_{2}}} d x+C\left(\|\nabla \Phi\|_{L^{2+\beta_{1}}}^{2+\beta_{1}}+\sum_{i=1,2}\left\|\nabla p_{i}\right\|_{L^{2-\beta_{2}}}^{1-\beta_{2}}\|\nabla \Phi\|_{L^{2-\beta_{2}}}+\sum_{i=1,2}\left\|\bar{p}_{i}\right\|\left\|\Phi_{t}\right\|\right)$.

Thus,

$$
\frac{d}{d t}\|\bar{P}(t)\|^{2} \leq-2 C_{1} \int_{U} \frac{|\nabla \bar{P}|^{2+\beta_{1}}}{\omega^{\beta_{1}+\beta_{2}}} d x+C\left(\Lambda(t)^{1-\beta_{2}}+\left\|\bar{p}_{1}(t)\right\|+\left\|\bar{p}_{2}(t)\right\|\right) D(t) .
$$

Applying Hölder's inequality with powers $\frac{2+\beta_{1}}{2-\beta_{2}}$ and $\frac{2+\beta_{1}}{\beta_{1}+\beta_{2}}$, we have

$\int_{U}|\nabla \bar{P}|^{2-\beta_{2}} d x=\int_{U} \frac{|\nabla \bar{P}|^{2-\beta_{2}}}{\omega^{\frac{\left(\beta_{1}+\beta_{2}\right)\left(2-\beta_{2}\right)}{2+\beta_{1}}}} \cdot \omega^{\frac{\left(\beta_{1}+\beta_{2}\right)\left(2-\beta_{2}\right)}{2+\beta_{1}}} d x \leq\left(\int_{U} \frac{|\nabla \bar{P}|^{2+\beta_{1}}}{\omega^{\beta_{1}+\beta_{2}}} d x\right)^{\frac{2-\beta_{2}}{2+\beta_{1}}}\left(\int_{U} \omega^{2-\beta_{2}} d x\right)^{\frac{\beta_{1}+\beta_{2}}{2+\beta_{1}}}$.

This implies

$$
\Lambda(t)^{\beta_{1}+\beta_{2}} \int_{U} \frac{|\nabla \bar{P}|^{2+\beta_{1}}}{\omega^{\beta_{1}+\beta_{2}}} d x \geq\left(\int_{U}|\nabla \bar{P}|^{2-\beta_{2}} d x\right)^{\frac{2+\beta_{1}}{2-\beta_{2}}}
$$


Combining (4.10) with Poincaré-Sobolev inequality (2.34) yields

$$
\int_{U} \frac{|\nabla \bar{P}|^{2+\beta_{1}}}{\omega^{\beta_{1}+\beta_{2}}} d x \geq C_{\mathrm{PS}}^{-\left(2+\beta_{1}\right)} \Lambda(t)^{-\left(\beta_{1}+\beta_{2}\right)}\left(\int_{U} \bar{P}^{2} d x\right)^{\frac{2+\beta_{1}}{2}} .
$$

Using (4.11) to estimate the first integral on the RHS of (4.9), we obtain for all $t>0$ that

$$
\frac{d}{d t}\|\bar{P}(t)\|^{2} \leq-C_{2} \Lambda(t)^{-\left(\beta_{1}+\beta_{2}\right)}\|\bar{P}(t)\|^{2+\beta_{1}}+C\left(\Lambda(t)^{1-\beta_{2}}+\left\|\bar{p}_{1}(t)\right\|+\left\|\bar{p}_{2}(t)\right\|\right) D(t),
$$

where $C_{2}=2 C_{1} C_{\mathrm{PS}}^{-\left(2+\beta_{1}\right)}$. Denote $y(t)=\|\bar{P}(t)\|^{2}$ and rewrite (4.12) as

$$
\frac{d y}{d t} \leq-C_{2} \Lambda(t)^{-\left(\beta_{1}+\beta_{2}\right)} y(t)^{\frac{2+\beta_{1}}{2}}+C\left(\Lambda(t)^{1-\beta_{2}}+\left\|\bar{p}_{1}(t)\right\|+\left\|\bar{p}_{2}(t)\right\|\right) D(t) .
$$

Applying (3.7) in Lemma 3.2 to (4.13) proves (4.3). Similarly, applying (3.8) in Lemma 3.2 to (4.13), we obtain (4.4).

Next, we combine Proposition 4.1 with results in section 3 to derive more specific estimates.

According to (3.9),

$$
1+\left\|\bar{p}_{1}\right\|+\left\|\bar{p}_{2}\right\| \leq \mathcal{X}(t)
$$

where

$$
\mathcal{X}(t)=1+\sum_{i=1,2}\left\{\left\|\bar{p}_{i}(0)\right\|+\left(\operatorname{Env} f\left[\Psi_{i}\right](t)\right)^{\frac{1}{2-\beta_{2}}}\right\} .
$$

By (3.34) and (3.58),

$$
\Lambda(t) \leq C \hat{\mathcal{Y}}(t)^{\frac{1}{2-\beta_{2}}}
$$

where

$$
\hat{\mathcal{Y}}(t)=\left\{\begin{array}{rlrl}
1 & +\sum_{i=1,2}\left(\left\|\bar{p}_{i}(0)\right\|^{2}+e^{-\frac{t}{2}}\left\|\nabla p_{i}(0)\right\|_{L^{2-\beta_{2}}}^{2-\beta_{2}}\right. & \\
& \left.+\left(\operatorname{Envf}\left[\Psi_{i}\right](t)\right)^{\frac{2}{2-\beta_{2}}}+\int_{0}^{t} e^{-\frac{1}{2}(t-\tau)}\left\|\nabla \Psi_{i, t}(\tau)\right\|^{2} d \tau\right) & & \\
1+\sum_{i=1,2}\left(\left\|\bar{p}_{i}(0)\right\|^{2}+\left(\operatorname{Envf}\left[\Psi_{i}\right](t)\right)^{\frac{2}{2-\beta_{2}}}+\int_{t-1}^{t}\left\|\nabla \Psi_{i, t}(\tau)\right\|^{2} d \tau\right) & & \text { if } t \geq 1 .
\end{array}\right.
$$

To simplify expressions of our estimates, we set

$$
\mathcal{Y}_{0}=1+\sum_{i=1,2}\left(\left\|\bar{p}_{i}(0)\right\|^{2}+\left\|\nabla p_{i}(0)\right\|_{L^{2-\beta_{2}}}^{2-\beta_{2}}\right)
$$

and define the function

$$
\tilde{\mathcal{Y}}(t)=\mathcal{Y}_{0}+\sum_{i=1,2}\left(\operatorname{Envf}\left[\Psi_{i}\right](t)\right)^{\frac{2}{2-\beta_{2}}}+ \begin{cases}\int_{0}^{t} e^{-\frac{1}{2}(t-\tau)} \sum_{i=1,2}\left\|\nabla \Psi_{i, t}(\tau)\right\|^{2} d \tau & \text { if } 0 \leq t<1 \\ \int_{t-1}^{t} \sum_{i=1,2}\left\|\nabla \Psi_{i, t}(\tau)\right\|^{2} d \tau & \text { if } t \geq 1 .\end{cases}
$$

Then $\hat{\mathcal{Y}}(t) \leq \tilde{\mathcal{Y}}(t)$ and $\mathcal{X}(t) \leq \tilde{\mathcal{Y}}^{1 / 2}(t)$. These properties and 4.14), 4.15) imply

$$
\begin{gathered}
\Lambda(t) \leq C \tilde{\mathcal{Y}}(t)^{\frac{1}{2-\beta_{2}}} \\
\Lambda(t)^{1-\beta_{2}}+\left\|\bar{p}_{1}(t)\right\|+\left\|\bar{p}_{2}(t)\right\| \leq C\left(\widetilde{\mathcal{Y}}(t)^{\frac{1-\beta_{2}}{2-\beta_{2}}}+\tilde{\mathcal{Y}}(t)^{1 / 2}\right) \leq C \widetilde{\mathcal{Y}}(t)^{1 / 2} .
\end{gathered}
$$


Above, we used the fact $1 / 2>\left(1-\beta_{2}\right) /\left(2-\beta_{2}\right)$ and $\widetilde{\mathcal{Y}}(t) \geq 1$.

For asymptotic estimates, we will use the following numbers

$$
\begin{aligned}
\widetilde{\mathcal{A}} & =\left(\sum_{i=1,2} \limsup _{t \rightarrow \infty} f\left[\Psi_{i}\right](t)\right)^{\frac{1}{2-\beta_{2}}}, \quad \widetilde{\mathcal{K}}=\widetilde{\mathcal{A}}^{2}+\sum_{i=1,2} \limsup _{t \rightarrow \infty} \int_{t-1}^{t}\left\|\nabla \Psi_{i, t}(\tau)\right\|^{2} d \tau, \\
\mathcal{D} & =\limsup _{t \rightarrow \infty} D(t) .
\end{aligned}
$$

Now we can estimate the $L^{2}$-norm of $\bar{P}(t)$ utterly in terms of the initial and boundary data.

Theorem 4.2. For $t \geq 0$,

$$
\|\bar{P}(t)\|^{2} \leq\|\bar{P}(0)\|^{2}+C\left\{\operatorname{Env}\left[\widetilde{\mathcal{Y}}(t)^{\frac{\beta_{1}+\beta_{2}}{2-\beta_{2}}+\frac{1}{2}} D(t)\right]\right\}^{\frac{2}{2+\beta_{1}}} .
$$

If $\widetilde{\mathcal{K}}<\infty$ then

$$
\limsup _{t \rightarrow \infty}\|\bar{P}(t)\|^{2} \leq C\left\{(1+\widetilde{\mathcal{K}})^{\frac{\beta_{1}+\beta_{2}}{2-\beta_{2}}+\frac{1}{2}} \mathcal{D}\right\}^{\frac{2}{2+\beta_{1}}}
$$

Proof. It follows from (4.3), (4.18) and (4.19) that

$$
\|\bar{P}(t)\|^{2} \leq\|\bar{P}(0)\|^{2}+C\left\{\operatorname{Env}\left[\tilde{\mathcal{Y}}(t)^{\frac{\beta_{1}+\beta_{2}}{2-\beta_{2}}} \tilde{\mathcal{Y}}(t)^{\frac{1}{2}} D(t)\right]\right\}^{\frac{2}{2+\beta_{1}}}
$$

which implies (4.21).

We have from limit estimates (3.68) and (3.11) that

$$
\limsup _{t \rightarrow \infty} \Lambda(t) \leq C(1+\widetilde{\mathcal{K}})^{\frac{1}{2-\beta_{2}}}, \quad \limsup _{t \rightarrow \infty}\left(1+\left\|\bar{p}_{1}(t)\right\|+\left\|\bar{p}_{2}(t)\right\|\right) \leq C(1+\widetilde{\mathcal{A}}) \leq C(1+\widetilde{\mathcal{K}})^{\frac{1}{2}} .
$$

Combining (4.23) with (4.4) we obtain

$$
\limsup _{t \rightarrow \infty}\|\bar{P}(t)\|^{2} \leq C\left\{(1+\widetilde{\mathcal{K}})^{\frac{\beta_{1}+\beta_{2}}{2-\beta_{2}}}\left[(1+\widetilde{\mathcal{K}})^{\frac{1}{2}}+(1+\widetilde{\mathcal{K}})^{\frac{1-\beta_{2}}{2-\beta_{2}}}\right] \mathcal{D}\right\}^{\frac{2}{2+\beta_{1}}}
$$

Note that $1 / 2>\left(1-\beta_{2}\right) /\left(2-\beta_{2}\right)$, then (4.22) follows (4.24).

\section{$5 \quad$ Structural stability}

In this section, we consider the case $K(\xi)=K_{I}(\xi, \vec{a})$ in (1.23), and study the dependence of the solutions to IBVP (3.1) on the coefficient vector $\vec{a}$.

Let $N \geq 1$ and the exponent vector $\vec{\alpha}=\left(-\alpha, 0, \alpha_{1}, \ldots, \alpha_{N}\right)$ be fixed. Since $\vec{a}$ satisfies condition (1.21), we denote the set of admissible $\vec{a}$ by $S$, that is,

$$
S=\left\{\vec{a}=\left(a_{-1}, a_{0}, \ldots, a_{N}\right): a_{-1}, a_{N}>0, a_{0}, a_{1}, \ldots, a_{N-1} \geq 0\right\} .
$$

The following "perturbed monotonicity" is important for our structural stability in this section; it plays the same role as the monotonicity (Lemma 2.4) for the continuous dependence in section 4. Below, the notation $\vee$, resp. $\wedge$, denotes the maximum, resp. minimum, of two numbers or two vectors meaning coordinate-wise. 
Lemma 5.1 (Perturbed Monotonicity). Let $K_{I}(\xi, \vec{a})$ be defined as in (1.23). For any coefficient vectors $\vec{a}^{(1)}, \vec{a}^{(2)} \in S$, and any $y, y^{\prime} \in \mathbb{R}^{n}$, one has

$$
\begin{aligned}
\left(K_{I}\left(\left|y^{\prime}\right|, \vec{a}^{(1)}\right) y^{\prime}-K_{I}\left(|y|, \vec{a}^{(2)}\right) y\right) & \cdot\left(y^{\prime}-y\right) \geq \frac{d_{6}\left|y-y^{\prime}\right|^{2+\beta_{1}}}{\left(1+|y|+\left|y^{\prime}\right|\right)^{\beta_{1}+\beta_{2}}} \\
& -d_{7} K\left(|y| \vee\left|y^{\prime}\right|, \vec{a}^{(1)} \wedge \vec{a}^{(2)}\right)\left(|y| \vee\left|y^{\prime}\right|\right)\left|\vec{a}^{(1)}-\vec{a}^{(2)}\right|\left|y-y^{\prime}\right|,
\end{aligned}
$$

where $d_{6}=d_{6}\left(\vec{a}^{(1)}, \vec{a}^{(2)}\right)$ and $d_{7}=d_{7}\left(\vec{a}^{(1)}, \vec{a}^{(2)}\right)$ are positive constants defined by

$$
\begin{aligned}
& d_{6}=\frac{1-\beta_{2}}{\left(\beta_{1}+1\right)\left[2(N+2) \max \left\{1, a_{i}^{(j)}: i=-1,0, \ldots, N, j=1,2\right\}\right]^{\beta_{1}+1}}, \\
& d_{7}=\frac{N+1}{(1-\alpha) \min \left\{a_{-1}^{(1)}, a_{-1}^{(2)}, a_{N}^{(1)}, a_{N}^{(2)}\right\}} .
\end{aligned}
$$

Proof. Let $\vec{a}^{(1)}, \vec{a}^{(2)} \in S$ and $y, y^{\prime} \in \mathbb{R}^{n}$. Same as in Lemma 2.4, it suffices to consider the case when the line segment $\left[y, y^{\prime}\right]$ does not contain the origin. For $t \in[0,1]$, let

$$
\gamma(t)=t y+(1-t) y^{\prime}, \quad \vec{b}(t)=\left(b_{-1}(t), b_{0}(t), \ldots, b_{N}(t)\right) \stackrel{\text { def }}{=} t \vec{a}^{(1)}+(1-t) \vec{a}^{(2)},
$$

and define

$$
z(t)=K(|\gamma(t)|, \vec{b}(t)) \gamma(t) \cdot\left(y-y^{\prime}\right)
$$

We have

$$
I \stackrel{\text { def }}{=}\left[K\left(|y|, \vec{a}^{(1)}\right) y-K\left(\left|y^{\prime}\right|, \vec{a}^{(2)}\right) y^{\prime}\right] \cdot\left(y^{\prime}-y\right)=z(1)-z(0)=\int_{0}^{1} z^{\prime}(t) d t .
$$

In calculations below, we use the following short-hand notation for partial derivatives

$$
X_{s}=\partial X / \partial s, \quad X_{\xi}=\partial X / \partial \xi, \quad X_{a_{i}}=\partial X / \partial a_{i}, \text { and } X_{\vec{a}}=\partial X / \partial \vec{a} .
$$

Elementary calculations give

$$
I=\int_{0}^{1} h_{1}(t) d t+\int_{0}^{1} h_{2}(t) d t \stackrel{\text { def }}{=} I_{1}+I_{2},
$$

where

$$
\begin{aligned}
& h_{1}(t)=K(|\gamma(t)|, \vec{b}(t))\left|y-y^{\prime}\right|^{2}+K_{\xi}(|\gamma(t)|, \vec{b}(t)) \frac{\left|\gamma(t) \cdot\left(y-y^{\prime}\right)\right|^{2}}{|\gamma(t)|}, \\
& h_{2}(t)=K_{\vec{a}}(|\gamma(t)|, \vec{b}(t))\left(\vec{a}^{(1)}-\vec{a}^{(2)}\right) \gamma(t) \cdot\left(y-y^{\prime}\right) .
\end{aligned}
$$

- Estimation of $I_{1}$. By Lemma 2.2 ,

$$
K_{\xi}(|\gamma(t)|, \vec{b}(t)) \geq-\beta_{2} \frac{K(|\gamma(t)|, \vec{b}(t))}{|\gamma(t)|} .
$$

Same as the proof of (2.29),

$$
h_{1}(t) \geq \frac{d_{2}(t)\left(1-\beta_{2}\right)\left|y^{\prime}-y\right|^{2}}{\left(1+|y|+\left|y^{\prime}\right|\right)^{\beta_{1}+\beta_{2}}}|\gamma(t)|^{\beta_{1}},
$$


where

$$
d_{2}(t)=\frac{1}{\left(\max \left\{1, \sum_{i=-1}^{N} \vec{b}_{i}(t)\right\}\right)^{1+\beta_{1}}} .
$$

We can estimate $d_{2}(\vec{b}(t)) \geq d_{2}^{*}$ for all $t \in[0,1]$, where

$$
d_{2}^{*}=\frac{1}{\left((N+2) \max \left\{1, a_{i}^{(j)}: i=-1,0, \ldots, N, j=1,2\right\}\right)^{1+\beta_{1}}} .
$$

Hence, it follows (5.3) that

$$
\int_{0}^{1} h_{1}(t) d t \geq \frac{d_{2}^{*}\left(1-\beta_{2}\right)\left|y^{\prime}-y\right|^{2}}{\left(1+|y|+\left|y^{\prime}\right|\right)^{\beta_{1}+\beta_{2}}} \int_{0}^{1}|\gamma(t)|^{\beta_{1}} d t
$$

Same calculations in (2.31) and (2.32) of Lemma 2.4 show that

$$
\int_{0}^{1}|\gamma(t)|^{\beta_{1}} d t \geq \frac{\left|y^{\prime}-y\right|^{\beta_{1}}}{2^{\beta_{1}+1}\left(\beta_{1}+1\right)}
$$

It follows from (5.4) and (5.5) that

$$
I_{1}=\int_{0}^{1} h_{1}(t) d t \geq \frac{d_{6}\left|y-y^{\prime}\right|^{2+\beta_{1}}}{\left(1+|y|+\left|y^{\prime}\right|\right)^{\beta_{1}+\beta_{2}}} .
$$

- Estimation of $I_{2}$. We find the partial derivative of $K(\xi, \vec{a})$ in $\vec{a}$. In calculations below, we denote, for convenience, $\alpha_{-1}=-\alpha$.

For $i=-1,0,1, \ldots, N$, taking the partial derivative in $a_{i}$ of the identity $K(\xi, \vec{a})=1 / g(s(\xi, \vec{a}), \vec{a})$, we find that

$$
K_{a_{i}}(\xi, \vec{a})=-\frac{g_{a_{i}}+g_{s} \cdot s_{a_{i}}}{g^{2}}=-K(\xi, \vec{a}) \frac{g_{a_{i}}+g_{s} \cdot s_{a_{i}}}{g} .
$$

Similarly, from $s g(s, \vec{a})=\xi$, we have for $i=-1,0,1, \ldots, N$,

$$
s_{a_{i}} \cdot g+s \cdot\left(g_{a_{i}}+g_{s} \cdot s_{a_{i}}\right)=0,
$$

which implies

$$
s_{a_{i}}=\frac{-s \cdot g_{a_{i}}}{g+s \cdot g_{s}}
$$

Then we obtain

$$
K_{a_{i}}(\xi, \vec{a})=-K(\xi, \vec{a}) \frac{g_{a_{i}}+g_{s} \cdot \frac{-s \cdot g_{a_{i}}}{g+s \cdot g_{s}}}{g}=-K(\xi, \vec{a}) \frac{g_{a_{i}}}{g+s \cdot g_{s}}=-K(\xi, \vec{a}) \frac{s^{\alpha_{i}}}{g+s \cdot g_{s}},
$$

and consequently,

$$
\sum_{i=-1}^{N}\left|K_{a_{i}}(\xi, \vec{a})\right| \leq K(\xi, \vec{a}) \frac{s^{-\alpha}+1+s^{\alpha_{1}}+\cdots+s^{\alpha_{N}}}{(1-\alpha) a_{-1} s^{-\alpha}+a_{0}+\left(1+\alpha_{1}\right) a_{1} s^{\alpha_{1}}+\cdots+\left(1+\alpha_{N}\right) a_{N} s^{\alpha_{N}}} .
$$

Using (3.3), we have

$$
1, s^{\alpha_{1}}, \ldots, s^{\alpha_{N-1}} \leq s^{-\alpha}+s^{\alpha_{N}}
$$


Hence,

$$
\sum_{i=-1}^{N}\left|K_{a_{i}}(\xi, \vec{a})\right| \leq K(\xi, \vec{a}) \frac{(N+1)\left(s^{-\alpha}+s^{\alpha_{N}}\right)}{(1-\alpha) a_{-1} s^{-\alpha}+\left(1+\alpha_{N}\right) a_{N} s^{\alpha_{N}}} \leq d(\vec{a}) K(\xi, \vec{a}) .
$$

where

$$
d(\vec{a})=\frac{N+1}{\min \left\{(1-\alpha) a_{-1},\left(1+\alpha_{N}\right) a_{N}\right\}} .
$$

Thus,

$$
\left|K_{\vec{a}}(\xi, \vec{a})\right| \leq d(\vec{a}) K(\xi, \vec{a})
$$

Now, by estimate (5.8)

$$
\begin{aligned}
\left|h_{2}(t)\right| & \leq\left|K_{\vec{a}}(|\gamma(t)|, \vec{b}(t))\right| \cdot\left|\vec{a}^{(1)}-\vec{a}^{(2)}\right| \cdot|\gamma(t)| \cdot\left|y-y^{\prime}\right| \\
& \leq d(\vec{b}(t)) K(|\gamma(t)|, \vec{b}(t))|\gamma(t)| \vec{a}^{(1)}-\vec{a}^{(2)}|\cdot| y-y^{\prime} \mid .
\end{aligned}
$$

Since $a_{i}^{(j)}$ is positive for $i=-1, N$ and $j=1,2$, the number $d(\vec{b}(t))$, for all $t \in[0,1]$, can be bounded by

$$
d(\vec{b}(t)) \leq \frac{N+1}{\min \left\{(1-\alpha) a_{-1}^{(1)},(1-\alpha) a_{-1}^{(2)},\left(1+\alpha_{N}\right) a_{N}^{(1)},\left(1+\alpha_{N}\right) a_{N}^{(2)}\right\}} \leq d_{7} .
$$

Applying Corollary 2.3 to $m=1 \geq \beta_{2}$ gives that the function $\xi K(\xi, \vec{b}(t))$ is increasing in $\xi$. This, together with (5.9) and the fact $|\gamma(t)| \leq|y| \vee\left|y^{\prime}\right|$, yields

$$
\left|h_{2}(t)\right| \leq d_{7} K\left(|y| \vee\left|y^{\prime}\right|, \vec{b}(t)\right)\left(|y| \vee\left|y^{\prime}\right|\right)\left|\vec{a}^{(1)}-\vec{a}^{(2)}\right| \cdot\left|y-y^{\prime}\right|
$$

Note from (5.7) that $K(\xi, \vec{a})$ is decreasing in each $a_{i}$, hence

$$
K(\xi, \vec{b}(t)) \leq K\left(\xi, \vec{a}^{(1)} \wedge \vec{a}^{(2)}\right) .
$$

Therefore,

$$
\left|h_{2}(t)\right| \leq d_{7} K\left(|y| \vee\left|y^{\prime}\right|, \vec{a}^{(1)} \wedge \vec{a}^{(2)}\right)\left(|y| \vee\left|y^{\prime}\right|\right)\left|\vec{a}^{(1)}-\vec{a}^{(2)}\right|\left|y-y^{\prime}\right|,
$$

and consequently,

$$
I_{2} \geq-\int_{0}^{1}\left|h_{2}(t)\right| d t \geq-d_{7} K\left(|y| \vee\left|y^{\prime}\right|, \vec{a}^{(1)} \wedge \vec{a}^{(2)}\right)\left(|y| \vee\left|y^{\prime}\right|\right)\left|\vec{a}^{(1)}-\vec{a}^{(2)}\right|\left|y-y^{\prime}\right|
$$

Thus, we obtain (5.1) by combining (5.2), (5.6) and (5.11).

Let $\mathcal{R}$ be a compact subset of $S$ and let the boundary data $\psi(x, t)$ be fixed.

For $i=1,2$, let $\vec{a}^{(i)} \in \mathcal{R}$, and let $p_{i}(x, t)$ be the solution of (3.1) with $K=K\left(\xi, \vec{a}^{(i)}\right)$. Our goal is to estimate $p_{1}(x, t)-p_{2}(x, t)$ in terms of $\vec{a}^{(1)}-\vec{a}^{(2)}$.

We will use the results in section 3 for estimates of $p_{1}$ and $p_{2}$. Examining constants $d_{2}, d_{3}, d_{4}$ in section 2, and $d_{6}, d_{7}$ in Lemma 5.1, we see that they can be made dependent only on $N, \alpha, \alpha_{N}$, $\beta_{1}, \beta_{2}$ and the following constants

$$
\begin{aligned}
& \bar{c}_{\mathcal{R}}=\max \left\{a_{i}:-1 \leq i \leq N, \vec{a}=\left(a_{i}\right)_{i=-1}^{N} \in \mathcal{R}\right\}, \\
& \underline{c}_{\mathcal{R}}=\min \left\{a_{-1}, a_{N}: \vec{a}=\left(a_{i}\right)_{i=-1}^{N} \in \mathcal{R}\right\} .
\end{aligned}
$$


Consequently, the constants $C, C_{0}, C_{1}, \ldots$ in calculations and bounds in section 3 can be made dependent only on $N, \alpha, \alpha_{N}, \beta_{1}, \beta_{2}, \bar{c}_{\mathcal{R}}, \underline{c}_{\mathcal{R}}$ and $C_{\mathrm{PS}}$. Such dependence will also apply to the generic, positive constant $C$ in this section.

Let $\Psi$ be the extension of $\psi$ as in section 3.1. The calculations in section 4 , when used in this section, will correspond to $\Psi_{1}=\Psi_{2}=\Psi$.

Let $P=p_{1}-p_{2}$. We have

$$
\begin{aligned}
\frac{\partial P}{\partial t} & =\nabla \cdot\left(K\left(\left|\nabla p_{1}\right|, \vec{a}^{(1)}\right) \nabla p_{1}-K\left(\left|\nabla p_{2}\right|, \vec{a}^{(2)}\right) \nabla p_{2}\right) \quad \text { on } U \times(0, \infty), \\
P & =0 \quad \text { on } \Gamma \times(0, \infty) .
\end{aligned}
$$

Let $f(t)$ be defined by (3.10), $\Lambda(t)$ by (4.2), and $\mathcal{Y}_{0}$ by (4.16). We define, similar to (4.17), the function

$$
\mathcal{Y}(t)=\mathcal{Y}_{0}+(\operatorname{Env} f(t))^{\frac{2}{2-\beta_{2}}}+ \begin{cases}\int_{0}^{t}\left\|\nabla \Psi_{t}(\tau)\right\|^{2} d \tau & \text { if } 0 \leq t<1 \\ \int_{t-1}^{t}\left\|\nabla \Psi_{t}(\tau)\right\|^{2} d \tau & \text { if } t \geq 1\end{cases}
$$

and, similar to (4.20), the numbers

$$
\mathcal{A}=\limsup _{t \rightarrow \infty} f(t)^{\frac{1}{2-\beta_{2}}} \quad \text { and } \quad \mathcal{K}=\mathcal{A}^{2}+\limsup _{t \rightarrow \infty} \int_{t-1}^{t}\left\|\nabla \Psi_{t}(\tau)\right\|^{2} d \tau .
$$

\section{Theorem 5.2.}

(i) For $t \geq 0$, one has

$$
\int_{U}|P(x, t)|^{2} d x \leq \int_{U}|P(x, 0)|^{2} d x+C \operatorname{Env} \mathcal{Y}(t)^{\frac{2}{2-\beta_{2}}}\left|\vec{a}^{(1)}-\vec{a}^{(2)}\right|^{\frac{2}{2+\beta_{1}}}
$$

(ii) If $\mathcal{K}<\infty$ then

$$
\limsup _{t \rightarrow \infty} \int_{U}|P(x, t)|^{2} d x \leq C(1+\mathcal{K})^{\frac{2}{2-\beta_{2}}}\left|\vec{a}^{(1)}-\vec{a}^{(2)}\right|^{\frac{2}{2+\beta_{1}}}
$$

Proof. Multiplying equation (5.12) by $P$, integrating over $U$, and by integration by parts, we find that

$$
\frac{1}{2} \frac{d}{d t} \int_{U} P^{2} d x=-\int_{U}\left(K\left(\left|\nabla p_{1}\right|, \vec{a}^{(1)}\right) \nabla p_{1}-K\left(\left|\nabla p_{2}\right|, \vec{a}^{(2)}\right) \nabla p_{2}\right) \cdot\left(\nabla p_{1}-\nabla p_{2}\right) d x .
$$

By the perturbed monotonicity (5.1) of $K(\xi, \vec{a})$, we have

$$
\frac{1}{2} \frac{d}{d t} \int_{U} P^{2} d x \leq-d_{6} J+C\left|\vec{a}^{(1)}-\vec{a}^{(2)}\right| \int_{U} K\left(\left|\nabla p_{1}\right| \vee\left|\nabla p_{2}\right|, \vec{a}^{(1)} \wedge \vec{a}^{(2)}\right)\left(\left|\nabla p_{1}\right| \vee\left|\nabla p_{2}\right|\right)^{2} d x,
$$

where

$$
J=\int_{U} \frac{|\nabla P|^{2+\beta_{1}}}{\left(1+\left|\nabla p_{1}\right|+\left|\nabla p_{2}\right|\right)^{\beta_{1}+\beta_{2}}} d x .
$$

Using (2.5) with $m=2$ for the last integral of (5.16), we have

$$
\frac{1}{2} \frac{d}{d t} \int_{U} P^{2} d x \leq-d_{6} J+C\left|\vec{a}^{(1)}-\vec{a}^{(2)}\right| \int_{U}\left(\left|\nabla p_{1}\right|^{2-\beta_{2}}+\left|\nabla p_{2}\right|^{2-\beta_{2}}\right) d x
$$


In (5.17), estimating $J$ by (4.11) we have, same as (4.13),

$$
\frac{1}{2} \frac{d}{d t} \int_{U} P^{2} d x \leq-C_{1}\left(\int_{U} P^{2} d x\right)^{\frac{2+\beta_{1}}{2}} \Lambda(t)^{-\left(\beta_{1}+\beta_{2}\right)}+C\left|\vec{a}^{(1)}-\vec{a}^{(2)}\right| \Lambda(t)^{2-\beta_{2}},
$$

where $C_{1}=2 d_{6} C_{\mathrm{PS}}^{-\left(2+\beta_{1}\right)}$.

(i) Same as (4.18), there is $C_{2}>0$ such that $\Lambda(t) \leq C_{2} \mathcal{Y}(t)^{\frac{1}{2-\beta_{2}}}$. Hence, we find that

$$
\frac{1}{2} \frac{d}{d t} \int_{U} P^{2} d x \leq-C_{3}\left(\int_{U} P^{2} d x\right)^{\frac{2+\beta_{1}}{2}} \mathcal{Y}(t)^{-\frac{\beta_{1}+\beta_{2}}{2-\beta_{2}}}+C\left|\vec{a}^{(1)}-\vec{a}^{(2)}\right| \mathcal{Y}(t)
$$

for some $C_{3}>0$. Applying (3.7) of Lemma 3.2 to differential inequality (5.19), we obtain

$$
\int_{U}|P(x, t)|^{2} d x \leq \int_{U}|P(x, 0)|^{2} d x+C\left\{\left|\vec{a}^{(1)}-\vec{a}^{(2)}\right| \operatorname{Env} \mathcal{Y}(t)^{1+\frac{\beta_{1}+\beta_{2}}{2-\beta_{2}}}\right\}^{\frac{2}{2+\beta_{1}}},
$$

thus, (5.14) follows.

(ii) By the virtue of (3.68) and (5.13),

$$
\limsup _{t \rightarrow \infty} \Lambda(t) \leq 1+\sum_{i=1,2} \limsup _{t \rightarrow \infty}\left\|\nabla p_{i}\right\|_{L^{2-\beta_{2}}} \leq C(1+\mathcal{K})^{\frac{1}{2-\beta_{2}}}<\infty .
$$

Thus,

$$
\int_{0}^{\infty} \Lambda(t)^{-\left(\beta_{1}+\beta_{2}\right)} d t=\infty
$$

Applying Lemma 3.2 to (5.18), for $\theta=\frac{2+\beta_{1}}{2}$, we have

$$
\limsup _{t \rightarrow \infty} \int_{U} P^{2} d x \leq C\left[\left|\vec{a}^{(1)}-\vec{a}^{(2)}\right| \limsup _{t \rightarrow \infty} \Lambda(t)^{2+\beta_{1}}\right]^{\frac{2}{2+\beta_{1}}}=C\left|\vec{a}^{(1)}-\vec{a}^{(2)}\right|^{\frac{2}{2+\beta_{1}}} \limsup _{t \rightarrow \infty} \Lambda^{2}(t) .
$$

Therefore, we obtain (5.15) from (5.21) and (5.20).

\section{References}

[1] E. Aulisa, L. Bloshanskaya, L. Hoang, and A. Ibragimov. Analysis of generalized Forchheimer flows of compressible fluids in porous media. J. Math. Phys., 50(10):103102:44pp, 2009.

[2] J. Bear. Dynamics of Fluids in Porous Media. American Elsevier Pub. Co., New York, 1972.

[3] E. Celik and L. Hoang. Maximum estimates for generalized Forchheimer flows in heterogeneous porous media. 2015. submitted, preprint http://arxiv.org/abs/1510.09000.

[4] E. Celik and L. Hoang. Generalized Forchheimer flows in heterogeneous porous media. Nonlinearity, 29(3):1124-1155, 2016.

[5] E. Celik, L. Hoang, and T. Kieu. Generalized Forchheimer flows of isentropic gases. 2015. submitted, preprint http://arxiv.org/abs/1504.00742.

[6] E. Celik, L. Hoang, and T. Kieu. Doubly nonlinear parabolic equations for a general class of Forchheimer gas flows in porous media. 2016. submitted, preprint http://arxiv.org/abs/1601.00703. 
[7] E. DiBenedetto. Degenerate parabolic equations. Universitext. Springer-Verlag, New York, 1993.

[8] C. Dudgeon. Non-Darcy flow of groundwater. Part 1. Theoretical, experimental and numerical studies. Report No. 162. Water research laboratory, The University of New South Wales, 1985.

[9] P. Forchheimer. Wasserbewegung durch Boden. Zeit. Ver. Deut. Ing., 45:1781-1788, 1901.

[10] P. Forchheimer. Hydraulik. Number Leipzig, Berlin, B. G. Teubner. 1930. 3rd edition.

[11] L. Hoang and A. Ibragimov. Structural stability of generalized Forchheimer equations for compressible fluids in porous media. Nonlinearity, 24(1):1-41, 2011.

[12] L. Hoang and A. Ibragimov. Qualitative study of generalized Forchheimer flows with the flux boundary condition. Adv. Diff. Eq., 17(5-6):511-556, 2012.

[13] L. Hoang, A. Ibragimov, T. Kieu, and Z. Sobol. Stability of solutions to generalized Forchheimer equations of any degree. J. Math. Sci., 210(4):476-544, 2015.

[14] L. Hoang and T. Kieu. Interior estimates for generalized Forchheimer flows of slightly compressible fluids. 2014. submitted, preprint http://arxiv.org/abs/1404.6517.

[15] L. Hoang and T. Kieu. Global estimates for generalized Forchheimer flows of slightly compressible fluids. Journal d'Analyse Mathematique, 2015. accepted.

[16] L. T. Hoang, A. Ibragimov, and T. T. Kieu. One-dimensional two-phase generalized Forchheimer flows of incompressible fluids. J. Math. Anal. Appl., 401(2):921-938, 2013.

[17] L. T. Hoang, A. Ibragimov, and T. T. Kieu. A family of steady two-phase generalized Forchheimer flows and their linear stability analysis. J. Math. Phys., 55(12):123101:32pp, 2014.

[18] L. T. Hoang, T. T. Kieu, and T. V. Phan. Properties of generalized Forchheimer flows in porous media. J. Math. Sci., 202(2):259-332, 2014.

[19] D. Jerison and C. E. Kenig. The inhomogeneous Dirichlet problem in Lipschitz domains. J. Funct. Anal., 130(1):161-219, 1995.

[20] M. Muskat. The flow of homogeneous fluids through porous media. McGraw-Hill Book Company, inc., 1937.

[21] D. A. Nield and A. Bejan. Convection in porous media. Springer-Verlag, New York, fourth edition, 2013.

[22] F. Siddiqui, M. Y. Soliman, W. House, and A. Ibragimov. Pre-Darcy flow revisited under experimental investigation. Journal of Analytical Science and Technology, 7(2), 2016.

[23] J. Soni, N. Islam, and P. Basak. An experimental evaluation of non-Darcian flow in porous media. Journal of Hydrology, 38(3-4):231-241, 1978.

[24] B. Straughan. Stability and wave motion in porous media, volume 165 of Applied Mathematical Sciences. Springer, New York, 2008.

[25] J. L. Vázquez. The porous medium equation. Oxford Mathematical Monographs. The Clarendon Press Oxford University Press, Oxford, 2007. Mathematical theory. 